\title{
VUV photoemission studies of candidate Large Hadron Collider vacuum chamber materials
}

\author{
R. Cimino \\ INFN Sezione di Trieste, Trieste, Italy \\ I. R. Collins and V. Baglin \\ LHC Vacuum Group, CERN, Geneva, Switzerland \\ (Received 19 January 1999; published 30 June 1999)
}

\begin{abstract}
In the context of future accelerators and, in particular, the beam vacuum of the Large Hadron Collider (LHC), a $27 \mathrm{~km}$ circumference proton collider to be built at CERN, VUV synchrotron radiation (SR) has been used to study both qualitatively and quantitatively candidate vacuum chamber materials. Emphasis is given to show that angle and energy resolved photoemission is an extremely powerful tool to address important issues relevant to the LHC, such as the emission of electrons that contributes to the creation of an electron cloud which may cause serious beam instabilities and unmanageable heat loads on the cryogenic system. Here we present not only the measured photoelectron yields from the proposed materials, prepared on an industrial scale, but also the energy and in some cases the angular dependence of the emitted electrons when excited with either a white light (WL) spectrum, simulating that in the arcs of the LHC, or monochromatic light in the photon energy range of interest. The effects on the materials examined of WL irradiation and/or ion sputtering, simulating the SR and ion bombardment expected in the LHC, were investigated. The studied samples exhibited significant modifications, in terms of electron emission, when exposed to the WL spectrum from the BESSY Toroidal Grating Monochromator beam line. Moreover, annealing and ion bombardment also induce substantial changes to the surface thereby indicating that such surfaces would not have a constant electron emission during machine operation. Such characteristics may be an important issue to define the surface properties of the LHC vacuum chamber material and are presented in detail for the various samples analyzed. It should be noted that all the measurements presented here were recorded at room temperature, whereas the majority of the LHC vacuum system will be maintained at temperatures below $20 \mathrm{~K}$. The results cannot therefore be directly applied to these sections of the machine until measurements at cryogenic temperatures, i.e., in the presence of cryosorbed gas layers, are obtained. However, these results are directly relevant to all the warm regions of the LHC vacuum system, such as the experimental vacuum chambers and warm element vacuum chambers in the insertion regions. [S1098-4402(99)00040-3]
\end{abstract}

PACS numbers: 29.20.Dh, 07.30.Kf, 79.60.-i, 29.27.Bd

\section{INTRODUCTION}

The Large Hadron Collider (LHC) will provide two countercirculating proton beams with colliding energies of nominally $14 \mathrm{TeV}$ in the center of mass, requiring superconducting bending magnets operating in superfluid helium at $1.9 \mathrm{~K}$. In order to reduce the cryogenic power consumption at $1.9 \mathrm{~K}$ in the arcs, the heat load induced by the beam will be intercepted on a beam screen, which operates between 5 and $20 \mathrm{~K}$. In the arcs of the machine, molecules will be pumped through the pumping slots, distributed along the length of the beam screen, and trapped on the surrounding cold bore held at $1.9 \mathrm{~K}$.

The emitted synchrotron radiation (SR) from the circulating protons, with a critical energy of $44.1 \mathrm{eV}$, is a major consideration for the design of the vacuum system. Its radiated power induces a heat load of $0.2 \mathrm{~W} / \mathrm{m}$ per beam and may (i) stimulate gas desorption of weakly and tightly bound gases from the walls of the vacuum system either directly by photons or mediated by electrons $[1,2]$, (ii) create photoelectrons which can be accelerated, to an average energy of $380 \mathrm{eV}$ [3], towards the opposite wall by the positive space charge of the bunched beam leading to additional gas desorption and heat loads on the cryogenic system, (iii) create secondary electrons which may contribute to electron multipacting [2]. This latter phenomenon is a resonant effect where a cloud of secondary electrons oscillates, in phase with the bunched beam, between opposite walls of the vacuum chamber. Simulations performed by considering the maximum of the secondary electron yield (SY), $\delta$, as the representative parameter of the SY suggest that if $\delta$ exceeds a critical value the cloud may increase exponentially [3], leading to beam instabilities, unmanageable heat loads to the cryogenic system, and, ultimately, to beam loss.

In addition to the SR, ion induced desorption is another major consideration in the design of the LHC. Positive ions, created by ionization of the residual gas and repelled by the positive space charge of the beam, bombard the vacuum chamber wall and desorb tightly and weakly bound molecules that would lead to a pressure instability if careful design is not considered [2]. Consequently, significant modifications of the industrial ex situ prepared vacuum chambers can be expected during machine operation. Such surface cleaning induced by SR, ion bombardment, and electron bombardment may 
therefore result in changes in the electron yields as a function of machine operation.

Calculations based on simulations are in progress to estimate the cryogenic heat load due to photoelectrons and secondary electrons and, in doing so, provide information on the sensitivity of the heat loads and electron cloud to the input parameters $[3,4]$. It has been predicted that only the secondary electrons emitted with a kinetic energy in the range of 1 to $6 \mathrm{eV}$ play a role in multipacting. Realistic input values for the photoelectron yield (PY), the energy and angular distribution of the emitted electrons, the reflectivity, etc., from technical surfaces are required for design optimization.

SR photoemission is an ideal tool to provide this information. Preliminary experiments have been performed giving qualitative information [5]; here, quantitative information on the PY is presented. In addition, the detailed energy and angular distributions of the photoemitted electrons are presented. Data were acquired using both "white light" (WL) SR and monochromatic light as the excitation probe. Studies were performed to investigate the important issues of the cleaning effects of SR illumination and/or surface conditioning, such as ion bombardment or annealing.

It should be pointed out that the data presented here were collected with samples held at room temperature while the LHC beam screen was held at temperatures between 5 and $20 \mathrm{~K}$. In order to simulate closely the LHC beam screen conditions, it will be necessary to measure samples at cryogenic temperatures in order to study issues such as the effect of physisorbed gases on the PY. Nevertheless, the results presented here are relevant to the vacuum chambers in the room temperature sections of the LHC. SR from the arcs may irradiate, either directly or as a result of multiple reflections, such sections thus allowing the possibility of all the machine to be subject to electron multipacting.

Experimental details are given in Sec. II including information of each sample preparation, followed in Sec. III by the experimental results and discussion. A summary of the results and an outlook on future work will be presented in Sec. IV.

\section{EXPERIMENTAL}

The experimental section is divided into three parts describing the photon source used, the sample studied with their detailed sample preparation, and the details of the data acquisition.

\section{A. The photon source}

In the LHC, given the mass of the circulating particles and their energy, the SR critical energy is $44.1 \mathrm{eV}$, so that the maximum flux emitted by the machine is in the ultraviolet up to the soft $\mathrm{x}$-ray region. To simulate as accurately as possible such a spectrum one has either to run an electron accelerator so that the SR has the same critical energy [6] or to use the existing SR sources, which generally have a higher critical energy, i.e., higher photon flux in the hard x-ray region, and filter their emission through a beam line. The latter can be achieved by attenuating the high energy part of the spectrum by multiple reflections from the different optical elements or by using monochromatic light in the range of interest for LHC.

There are no existing beam lines designed to deliver monochromatic light and a WL spectrum identical to the one of the LHC. This study employed a Toroidal Grating Monochromator beam line (TGM7) [7] installed on the BESSY synchrotron light source, Berlin, an $805 \mathrm{MeV}$ electron storage ring. The optical layout of such a monochromator is shown in Fig. 1. The grating can either be used in diffraction mode or in reflection mode in order to produce monochromatic light or WL, respectively. It is important to estimate the photon flux to quantitatively analyze the observed WL irradiation effects and total PY measurements obtained from the different samples. The WL spectrum was calculated and measured experimentally for the optical layout of Fig. 1, when using the grating as a mirror (in its "zero order" position). The BESSY bending magnet photon flux and the transmission of each of the optical elements as a function of its material and angle of incidence have been calculated using the Lawrence Berkeley National Laboratory codes available on the Web [8]. The total irradiated area was estimated to be of the order of $1 \mathrm{~mm}^{2}$.

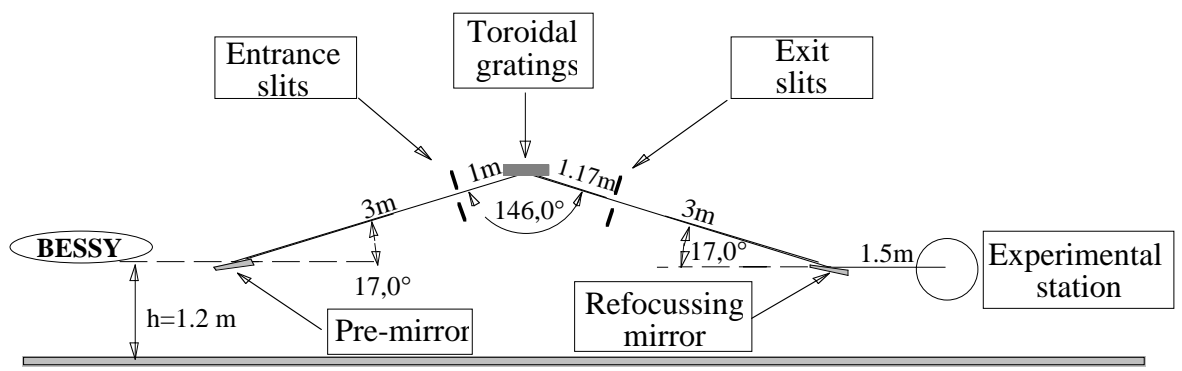

FIG. 1. TGM7 optical layout. 


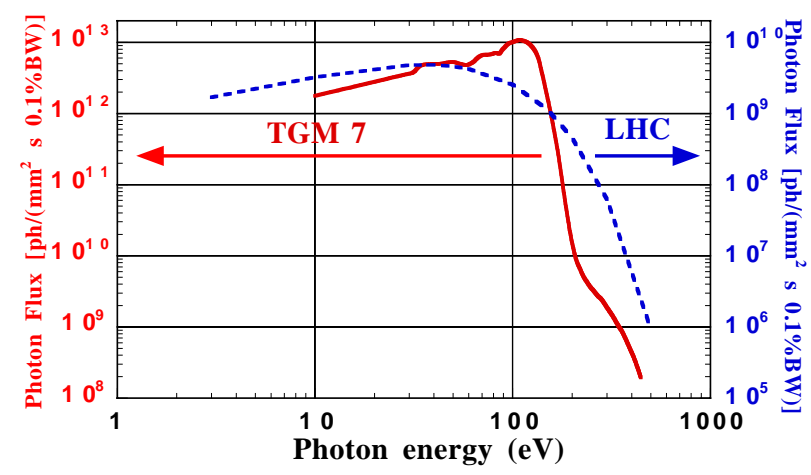

FIG. 2. (Color) A comparison between the LHC bending magnet photon flux with that obtained with the WL spectrum of the TGM7 beam line in BESSY. The integrated photon flux is $5.2 \times 10^{11}$ and $8.9 \times 10^{14}$ photons $/\left(\mathrm{s} \mathrm{mm}^{2}\right)$, respectively.

The so-calculated flux has been corrected by the angular acceptance of the beam line $(6 \mathrm{mrad}$ vertical $\times 15 \mathrm{mrad}$ horizontal) and the used slits sizes to give the spectrum shown in Fig. 2. The approximations made for this calculation are such that the spectrum in Fig. 2 gives an overestimated photon flux; i.e., losses due to imperfect alignment of the optical elements, etc., are not considered. However, the integrated flux of $8.9 \times 10^{14}$ photons $/\left(\mathrm{s} \mathrm{mm}^{2}\right)$ obtained from the calculated spectrum provides a consistent value with the actual photon flux measured experimentally from a clean gold reference sample (as shown in Sec. III D).

Figure 2 shows that the spectrum so obtained (having a cutoff mainly determined by the poor reflectivity of the optical elements at energies higher than $150 \mathrm{eV}$ ) is, in shape, a distorted replica of the LHC bending magnet spectrum. However, the number of photons $/\left(\mathrm{s} \mathrm{mm}^{2}\right)$ impinging on the sample is roughly 1000 times larger, per unit area, than that in the LHC. This considerably more intense photon flux was exploited to study the effects of high dose irradiation on the samples.

On the other hand, as shown in Table I, when using monochromatic light in the energy range between 18 and $110 \mathrm{eV}$, photon fluxes were comparable to that expected in the LHC for a mean value of BESSY beam currents which decayed from $700 \mathrm{~mA}$ at injection to $200 \mathrm{~mA}$ at the end of a run.
The flux reduction between WL and monochromatic light is not surprising since an efficiency loss of around a factor of 1000 is expected when using the grating in its diffraction mode. It should be pointed out that the photon dose has to be considered carefully when discussing WL electron emission spectrum (and measured total currents) since significant surface cleaning may occur and therefore may only be representative of strongly "irradiated samples."

\section{B. The data collection}

The samples (described in Sec. IIC) were installed via a fast load-lock into a UHV $\left(10^{-10}\right.$ mbar range) $\mu$-metal experimental chamber equipped with a VG ARUPS 500 angle-resolving electron spectrometer. The surfaces were characterized via constant final state (CFS) spectroscopy and angle-resolving photoemission using photon energies between 18 and $110 \mathrm{eV}$ spanning the $44.1 \mathrm{eV}$ critical energy spectrum of the LHC. In addition, the TGM7 WL spectrum was used to perform angleresolved photoemission measurements. The beam line slits define a photon resolution from 200 to $400 \mathrm{meV}$ for 18 to $110 \mathrm{eV}$ photon energy, respectively.

All the measurements were performed with the sample biased negatively with respect to ground to allow electrons with a very low kinetic energy to be measured accurately by the analyzer. The chosen bias of $-9.5 \mathrm{~V}$ (obtained from a battery) was sufficient to eliminate sample space charge problems and to permit one to measure the low energy part of the photoemission spectrum with the analyzer working in optimum conditions. Indeed, more than $-5 \mathrm{~V}$ bias produced spurious effects on the shape of the low kinetic energy secondaries due to the simultaneous collection of secondaries from the sample and from those created in the analyzer itself.

Slow electrons (with kinetic energies close to $0 \mathrm{eV}$ ) could be measured confidently in our setup due to the use of a $\mu$-metal UHV chamber that shielded efficiently stray magnetic fields acting near the sample. It is noted that even the Earth's magnetic field or spurious fields from ion pumps, etc., may affect strongly the detection of electrons with very low kinetic energies.

The samples were mounted on a partially electrically isolated sample holder (resistance to Earth was around

TABLE I. The measured monochromatic flux on the last refocusing gold mirror of the TGM7 beam line.

\begin{tabular}{ccccc}
\hline \hline $\begin{array}{c}\text { Photon } \\
\text { energy }(\mathrm{eV})\end{array}$ & $\begin{array}{c}I_{\text {mirror }} \\
(\mathrm{nA})\end{array}$ & $\begin{array}{c}\text { Au eff. } \\
{[9]}\end{array}$ & $\begin{array}{c}\text { Photon flux } \\
{\left[\text { photons } /\left(\mathrm{s} \mathrm{mm}^{2}\right) 0.1 \% \mathrm{bw}\right]}\end{array}$ & $\left.\begin{array}{c}\text { Error } \\
{\left[\text { photons } /\left(\mathrm{s} \mathrm{mm}^{2}\right)\right.}\end{array}\right) .1 \%$ bw] \\
\hline 20 & 0.08 & 0.091 & $2.5 \times 10^{9}$ & $1.3 \times 10^{9}$ \\
30 & 0.20 & 0.073 & $7.7 \times 10^{9}$ & $3.8 \times 10^{9}$ \\
50 & 0.50 & 0.057 & $2.5 \times 10^{9}$ & $1.3 \times 10^{9}$ \\
70 & 0.50 & 0.053 & $2.6 \times 10^{9}$ & $1.3 \times 10^{9}$ \\
100 & 0.40 & 0.034 & $3.3 \times 10^{9}$ & $1.7 \times 10^{9}$ \\
\hline \hline
\end{tabular}


$1 \mathrm{G} \Omega$ ). Such nonperfect isolation and the use of a negative $9.5 \mathrm{~V}$ bias allows us to measure accurately a sample drain current only with WL illumination (measured current was in $\mu \mathrm{A}$ range). It was impossible to measure with the required accuracy a sample drain current in the range of nA typical range when exciting with monochromatic light.

The photon flux, proportional to the drain current on the last gold plated refocusing mirror after the exit slit of the monochromator (Fig. 1), was measured during data acquisition. The measured drain currents were converted into photon flux using the quantum efficiency of clean gold, as cited in the literature [9]. Table I shows such data in the following way: in the first column is reported some selected photon energies for which the flux will be given; in the second column the mirror current reading is reported per $100 \mathrm{~mA}$ ring current; in the third column the Au efficiency values are shown [9]; in the fourth column the so-obtained flux values are given in units of photons $/\left(\mathrm{s} \mathrm{mm}^{2}\right) 0.1 \% \mathrm{bw}$; in the last column an estimated error of the quoted flux is given. Such errors are quite large and have been extracted taking into account the variation of the ring current in time, the difficulties to estimate correctly the spot size on the sample, etc. As stated in Sec. II A, the monochromatic photon fluxes depicted in Table I agree, within its error, with the required flux to be obtained to simulate the LHC spectrum shown in Fig. 2.

The WL photon flux estimation has been obtained by correcting the measured drain current from the last refocusing mirror by a weighted estimate of the energy dependent gold efficiency curve [9]. Such measured values are reported in Table III for different ring currents and agree well with the calculated WL integral flux [of $8.9 \times 10^{14}$ photons $\left./\left(\mathrm{s} \mathrm{mm}^{2}\right)\right]$ shown in Fig. 2 .

All measurements were performed with photons incident at $45^{\circ}$ and electrons emitted perpendicular to the surface, unless otherwise stated.

\section{The samples}

The samples studied can be divided into three major categories according to their relevance to the LHC. First, a set of $\mathrm{Cu}$-coated high-Mn content stainless steel samples was studied. Such a surface is now considered to be the base line design for the beam screen in the arcs [10]. Thus, it is relevant to study the properties of different surface preparations such that it can be optimized in terms of the resulting electron yield. The second category of samples is the so-called getter materials (such as TiZr and Pd, presently under development at CERN, and the commercial nonevaporable getter St 707) that are being considered on/in the vacuum chambers at room temperature to provide a means of distributed pumping. The third category is formed by alternative samples (such as $\mathrm{Al}$ alloy and TiN coated $\mathrm{Al}$ alloy, which is the solution adopted at PEP-II [11]). Such materials/coatings are being considered for the LHC experimental beam pipes due to their electrical properties and their relatively low transparency. Clean Au has been used as a well known and easy to reproduce reference.

The samples have been conditioned with different processes that are considered to be relevant to the LHC. First, all the getter materials, and some of the $\mathrm{Cu}$ based surfaces, have been heated to $250{ }^{\circ} \mathrm{C}-300{ }^{\circ} \mathrm{C}$, thereby simulating an in situ activation of the getters or a bakeout of the $\mathrm{Cu}$-coated surfaces. This conditioning is a possible preparation in the warm sections of the LHC but is not relevant in the arcs since it is not foreseen to bakeout the beam screen. Some of the samples were studied before and after argon ion sputtering, simulating the predicted ion bombardment in the LHC.

The samples studied are listed in Table II with a detailed description of their preparation to produce the analyzed surface. Sample $\mathrm{Cu}$ has already been measured in a previous experiment on a different beam line [5].

\section{RESULTS AND DISCUSSION}

The results obtained are divided into seven sections, each one addressing a particular key point: namely, (A) the photon energy dependence and angular distributions of the energy distribution curves (EDCs) of $\mathrm{Au}$, (B) the use of monochromatic SR to understand the effect of WL irradiations and surface conditioning effects, (C) the photon energy dependence of the PY (CFS), (D) the measured WL exposed PY of the as-received samples obtained from the sample drain current, (E) the measured WL exposed PY of the conditioned samples obtained from the sample drain current, $(F)$ the EDC of such WL exposed samples, $(\mathrm{G})$ the generation of "low dose" WL EDC, $(\mathrm{H})$ the energy distribution of the secondary electrons in the LHC.

\section{A. Photon energy dependence and angular distributions of the EDCs of Au}

The clean Au sample was studied in detail as it was necessary to study a well known and characterized sample in order to calibrate our experimental setup. All the electron energy analyzer calibrations, and most of the angleresolved studies, have been performed from this surface. It provided a reference sample from which estimates of confidence levels with which the data from the other studied samples could be treated. In Fig. 3 are shown a full series of EDCs from the clean $\mathrm{Au}$ as a function of photon energy. Valence band structures, Fermi edges, core levels, and secondary electron tails are clearly identified. The energy calibrations and resolutions can be deduced from the high energy cutoff (Fermi level). All EDCs were taken with a $20 \mathrm{~V}$ pass energy on the analyzer, which, together with the photon energy resolution, 
TABLE II. The sample and sample preparation details. The naming key will be used throughout the rest of the paper to refer to each sample studied.

\begin{tabular}{|c|c|c|}
\hline $\begin{array}{l}\text { Naming } \\
\text { key }\end{array}$ & Sample & Sample preparation \\
\hline $\mathrm{Au}$ & $\begin{array}{l}\text { Evaporated gold } \\
\text { Reference sample }\end{array}$ & $\begin{array}{l}\text { A clean } \mathrm{Au} \text { thin }(>5 \mu \mathrm{m}) \text { film was prepared by evaporation of pure } \mathrm{Au} \text {, by } \\
\text { resistive heating of } \mathrm{W} \text { crucible, onto a gold foil in } \mathrm{UHV}\left(p<3 \times 10^{-9} \text { mbar }\right) \text {. }\end{array}$ \\
\hline $\mathrm{Cu}$ & $\begin{array}{l}\text { Oxygen free electronic (OFE) } \\
\mathrm{Cu} \text { colaminated on } \\
\text { high-Mn content } \\
\text { stainless steel }\end{array}$ & $\begin{array}{l}\text { OFE Cu colaminated onto high-Mn content ( } 9 \% \text { wt) stainless steel (UNS21904) } \\
\text { and annealed at } 900{ }^{\circ} \mathrm{C} \text { for } 15 \text { min (from UGINE, France). It was chemically } \\
\text { cleaned by degreasing in perchloroethylene vapor at } 121{ }^{\circ} \mathrm{C} \text {, then deoxidizing } \\
\text { by immersion in an alkaline detergent, pickling in } \mathrm{HCl} \text {, passivation in a } \\
\text { chromic acid/ } \mathrm{H}_{2} \mathrm{SO}_{4} \text { bath, rinsing in demineralized water, and finally drying } \\
\text { with } \mathrm{N}_{2} \text { gas. The sample was held one year at atmospheric pressure. }\end{array}$ \\
\hline Cu-sp. & $\mathrm{Cu}$ sputtered & $\begin{array}{l}\text { A "clean" } \mathrm{Cu} \text { sample was obtained by in situ } 2 \mathrm{keV} \mathrm{Ar}^{+} \text {sputtering } \\
\left(P=1 \times 10^{-5} \mathrm{mbar}\right) \text { for } 30 \mathrm{~min} \text { of the } \mathrm{Cu} \text { sample. }\end{array}$ \\
\hline $\mathrm{Cu}-\mathrm{ab}$. & $\mathrm{Cu}$ air baked & $\begin{array}{l}\text { OFE Cu colaminated onto high-Mn content ( } 9 \% \text { wt) stainless steel (UNS21904) } \\
\text { and annealed at } 920^{\circ} \mathrm{C} \text { for } 7.5 \text { min under } \mathrm{H}_{2} \text { (from UGINE, France). It was } \\
\text { chemically cleaned by degreasing in perchloroethylene vapor at } 121{ }^{\circ} \mathrm{C} \text {, then } \\
\text { deoxidizing by immersion in an alkaline detergent, pickling in } \mathrm{HCl} \text {, passi- } \\
\text { vation in a chromic acid/ } \mathrm{H}_{2} \mathrm{SO}_{4} \text { bath, rinsing in demineralized water, and } \\
\text { finally drying with } \mathrm{N}_{2} \text { gas. Baked at } 350{ }^{\circ} \mathrm{C} \text { for } 24 \mathrm{~h} \text {, vented to air while at } \\
350^{\circ} \mathrm{C} \text { for } 15 \text { min, evacuated at } 350{ }^{\circ} \mathrm{C} \text {, and cooled to RT. The sample } \\
\text { was held } 3 \text { months at atmospheric pressure. }\end{array}$ \\
\hline $\mathrm{TiN}$ & TiN on extruded $\mathrm{Al}$ alloy & $\begin{array}{l}6063 \mathrm{Al} \text { alloy }(0.5 \% \mathrm{Mg}, 0.5 \% \mathrm{Si}) \text { and } 187 \mathrm{~nm} \mathrm{TiN} \mathrm{(DC} \mathrm{reactive} \mathrm{sputtered,} \\
\text { Ti cathode, } 20 \% \mathrm{~N} / \mathrm{Ar}, 170 \mathrm{mbar} \text { total pressure). }\end{array}$ \\
\hline TiN-sp. & TiN sputtered & $\begin{array}{l}\text { In situ } 2 \mathrm{keV} \mathrm{Ar}^{+} \text {sputtering }\left(P=1.7 \times 10^{-5} \mathrm{mbar}\right) \text { for } 5 \mathrm{~min} \text { of the } \mathrm{TiN} \\
\text { sample. }\end{array}$ \\
\hline $\mathrm{TiZr}$ & $\sim 1 \mu \mathrm{m} \mathrm{TiZr}$ on $\mathrm{OFE} \mathrm{Cu}$ & $\begin{array}{l}\sim 1 \mu \mathrm{m} \text { TiZr film deposited onto OFE Cu after having first removed } 120 \mu \mathrm{m} \\
\text { of } \mathrm{Cu} \text { by electropolishing then another } 10 \mu \mathrm{m} \text { as a result of a chemical } \\
\text { treatment in sulfamic acid }(5 \mathrm{~g} / 1), \text { ammonium citrate }(1 \mathrm{~g} / \mathrm{l}), \mathrm{n} \text {-butanol } \\
(50 \mathrm{ml} / \mathrm{l}) \text {, and } 30 \% \text { vol } \mathrm{H}_{2} \mathrm{O}_{2}(50 \mathrm{ml} / \mathrm{l}) \text {. }\end{array}$ \\
\hline TiZr-ac. & Activated TiZr with Procedure A & $\begin{array}{l}\text { Annealing of TiZr in UHV (pressure during annealing was less than } 10^{-8} \text { mbar) } \\
\left.\text { at }(330 \pm 30)^{\circ} \mathrm{C} \text { for } 4 \mathrm{~h} \text { (Procedure } \mathrm{A}\right) \text {. }\end{array}$ \\
\hline TiZr II & $\sim 1 \mu \mathrm{m} \mathrm{TiZr}$ on OFE $\mathrm{Cu}$ & A second identical TiZr sample. \\
\hline TiZr II-ac. & Activated TiZr with Procedure B & $\begin{array}{l}\text { Annealing of TiZr II in UHV (pressure during annealing was less than } \\
5 \times 10^{-9} \text { mbar) at }(330 \pm 30)^{\circ} \mathrm{C} \text { for } 2.25 \mathrm{~h} \text { (Procedure B). }\end{array}$ \\
\hline Cu-el. & $\mathrm{Cu}$ electropolished & OFE $\mathrm{Cu}$ electropolished (150 $\mu \mathrm{m}$ removed). \\
\hline Cu-el.-an. & $\begin{array}{l}\text { Annealed electropolished } \mathrm{Cu} \\
\text { with Procedure } \mathrm{B}\end{array}$ & $\begin{array}{l}\text { Annealing of Cu-el. sample in UHV (pressure during annealing was less than } \\
5 \times 10^{-9} \text { mbar) at }(330 \pm 30)^{\circ} \mathrm{C} \text { for } 2.25 \mathrm{~h} \text { (Procedure B). }\end{array}$ \\
\hline $\mathrm{Pd}$ & $0.3 \mu \mathrm{m}$ Pd on OFE $\mathrm{Cu}$ & $\begin{array}{l}0.3 \mu \mathrm{m} \text { Pd film deposited onto OFE } \mathrm{Cu} \text { after having first removed } 120 \mu \mathrm{m} \text { of } \\
\mathrm{Cu} \text { by electropolishing then another } 10 \mu \mathrm{m} \text { as a result of a chemical } \\
\text { treatment in sulfamic acid }(5 \mathrm{~g} / 1), \text { ammonium citrate }(1 \mathrm{~g} / \mathrm{l}), \mathrm{n} \text {-butanol } \\
(50 \mathrm{ml} / \mathrm{l}) \text {, and } 30 \% \text { vol } \mathrm{H}_{2} \mathrm{O}_{2}(50 \mathrm{ml} / \mathrm{l}) \text {. }\end{array}$ \\
\hline Pd-an. & Annealed Pd with Procedure C & $\begin{array}{l}\text { Annealing of Pd sample in UHV (pressure during annealing was less than } \\
\left.5 \times 10^{-9} \text { mbar) at }(300 \pm 30)^{\circ} \mathrm{C} \text { for } 8 \mathrm{~h} \text { (Procedure } \mathrm{C}\right) \text {. }\end{array}$ \\
\hline St 707 & St $707^{\mathrm{TM}}$ from SAES getters & $\begin{array}{l}\text { St } 707^{\mathrm{TM}}(\mathrm{Zr} 70 \%, \mathrm{~V} 24.6 \%, \text { Fe } 5.4 \%) \text { as described by the SAES getters data } \\
\text { sheet. }\end{array}$ \\
\hline St 707-ac. & $\begin{array}{l}\text { Activated St } 707^{\mathrm{TM}} \text { with } \\
\text { Procedure C }\end{array}$ & $\begin{array}{l}\text { Annealing of St } 707^{\mathrm{TM}} \text { sample in UHV (pressure during annealing was less than } \\
5 \times 10^{-9} \text { mbar) at }(300 \pm 30)^{\circ} \mathrm{C} \text { for } 8 \mathrm{~h} \text { (Procedure } \mathrm{C} \text { ). }\end{array}$ \\
\hline $\mathrm{Al}$ & Al alloy & $\begin{array}{l}\text { Aluminum alloy AA6060, degreased with perchloroethylene vapor at } 121^{\circ} \mathrm{C} \text {, } \\
\text { etched with alkaline detergent, and rinsed. }\end{array}$ \\
\hline Al-sp. & Al sputtered & In situ $2 \mathrm{keV} \mathrm{Ar}^{+}$sputtering $\left(P=1.7 \times 10^{-5} \mathrm{mbar}\right)$ for $5 \mathrm{~min}$ of $\mathrm{Al}$ sample. \\
\hline
\end{tabular}




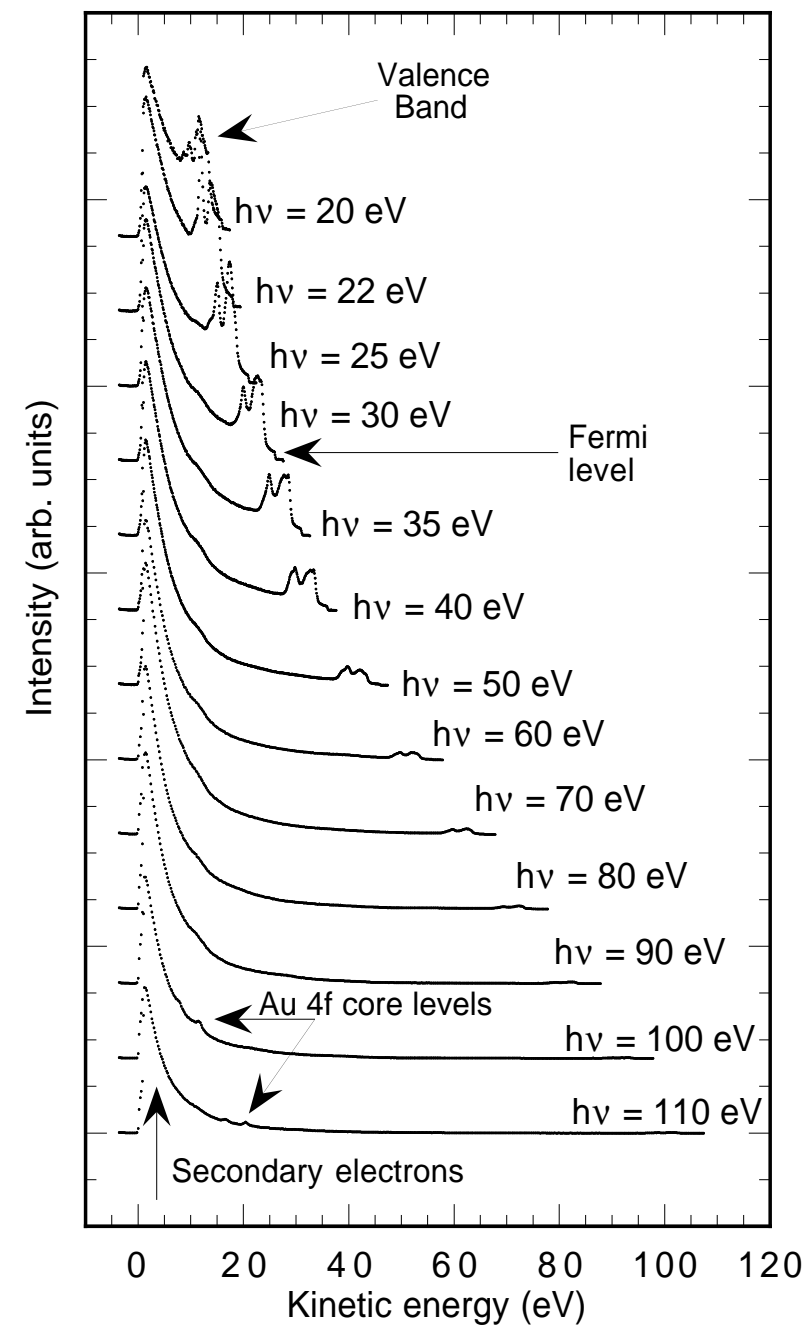

FIG. 3. A complete series of EDCs taken from the Au sample as a function of photon energy. Each EDC is shifted vertically for clarity.

correspond to a total electron energy resolution (FWHM) ranging from 0.2 to $0.4 \mathrm{eV}$ for 20 and $110 \mathrm{eV}$ photon energies, respectively. It should be noted that the onset of the secondary tail is directly related to the material work function (WF). The known clean polycrystalline $\mathrm{Au} \mathrm{WF}$ of $5.1 \mathrm{eV}$ [12] can then be used to extract WFs for the different samples studied. The WF is an important parameter since it can be simply related to PY. A high (low) WF material is expected to have a low (high) PY, giving a simple rule of thumb to predict the relevant behavior of a surface in terms of PY. We will discuss this aspect in more detail in a future publication.

Angle-resolved EDCs of the valence band electrons from clean $\mathrm{Au}$ are shown in Fig. 4. Similar data were recorded from some of the other samples but are not shown here. In the inset of this figure the energy integrated intensity is plotted as a function of angle, exhibiting a rather flat maximum around $20^{\circ}$ off normal emission, and, then, decreases with angle. Very probably there exist some systematic errors such that the maximum of

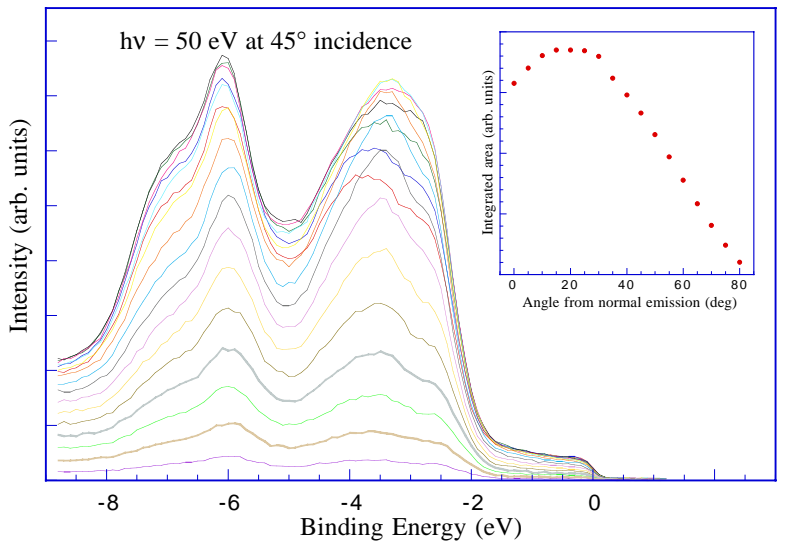

FIG. 4. (Color) The angle-resolved valence band photoemission spectra from clean Au taken with $50 \mathrm{eV}$ photons, referenced to the Fermi level. The energy integrated intensity as a function of emission angle is shown in the inset.

the distribution is not at normal emission, as one might expect. At this point these data serve to demonstrate that photoemission can provide both information on the intensity of the photoemitted electrons versus energy and emission angle. This information may be useful for the modeling of photoemitted electrons in the LHC and may be implemented in the simulations. In the LHC the electrons will be photoemitted mostly in the presence of a strong dipole field (8.4 T), so that their energy and angular distributions may play a role in the generated heat loads and beam perturbations due to the electron cloud.

\section{B. Understanding the effects of WL irradiation and surface conditioning}

As discussed in Sec. II A the number of photons/ ( $\mathrm{mm}^{2} 0.1 \%$ bandwidth) impinging on the sample with monochromatic light is comparable to that on the beam screen for the SR of the LHC bending magnet, whereas the TGM7 WL spectrum is some 1000 times more intense. This high photon flux thus corresponds to 5 to $50 \mathrm{~h}$ of LHC irradiation $\left[10^{16}-10^{17}\right.$ photons $\left./\left(\mathrm{s} \mathrm{mm}^{2}\right)\right]$.

One major advantage when using monochromatic light is therefore the substantially reduced photon flux with respect to the WL flux thereby simulating the initial conditions in the LHC. The monochromated light can be used efficiently as a tool to monitor and follow changes due to cleaning effects, induced by intense WL irradiation, ion bombardment, or heating, without significantly modifying the surface by the measurement itself.

The following is divided into sections for a selection of the samples studied.

\section{Photoemission with monochromatic light: $\mathrm{Cu}$}

$\mathrm{Cu}$ is currently the base line design material for the surface of the LHC beam screen. The resistivity of the vacuum chamber wall material is an important design 
criteria for the LHC. The image currents of the beam flowing in the surface of the material (over a given skin depth) lead to a beam impedance and a heat load on the cryogenic system. In fact, it is proposed to coat the beam screen, fabricated from a high-Mn content stainless steel, with $50 \mu \mathrm{m}$ of $\mathrm{Cu}$.

Photoemission spectra, taken with a photon energy of $30 \mathrm{eV}$, from the $\mathrm{Cu}$ sample as a function of conditioning with WL and $\mathrm{Ar}$ ion sputtering (sample $\mathrm{Cu}$-sp.), are shown in Fig. 5.

It is important to notice that the shape and the electron energy distribution of the as-received spectrum is structureless apart from the existence of a very high emission of secondary electrons at low kinetic energies. The asreceived EDC is similar to previous measurements performed on the same sample using a different experimental configuration (beam line and analyzer) [5].

Dramatic changes of the emitted electrons, both in the low energy part (mainly secondary electrons) and in the region closer to the Fermi energy (which is a distorted replica of the valence band density of states), are evident as a function of conditioning. The WL irradiation $\left(10^{16}-10^{17}\right.$ photons $\left./ \mathrm{mm}^{2}\right)$ partially cleans the dirty surface of the as-received sample, but does not clean it completely since the resulting spectrum is somewhat different from that exhibited by the essentially clean $\mathrm{Cu}$ surface, obtained by argon sputtering of the $\mathrm{Cu}$ sample. Ar ion sputtering (estimated dose $8 \times 10^{6} \mathrm{Ar}^{+} \mathrm{cm}^{-2}$ ) of $\mathrm{Cu}$ produces a nearly atomically clean copper surface, as confirmed by the clear appearance, in the valence band part of the spectrum, of a Fermi edge and the $\mathrm{Cu} 3 d$ bands at about $3 \mathrm{eV}$ below it. The spectrum is similar to the one published in the literature for clean polycrystalline copper [13]. The WL exposure of this Ar sputtered surface does not change the measured spectrum (data not shown for clarity) confirming that the WL has an effect only in the presence of a contaminant covered overlayer.

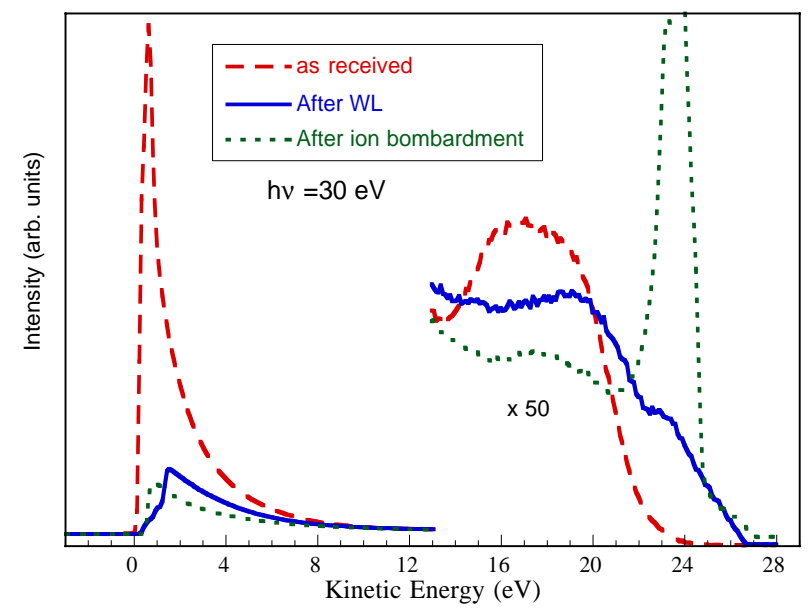

FIG. 5. (Color) The photoemission spectra, taken at $30 \mathrm{eV}$ photon energy, of $\mathrm{Cu}$ as received, after WL exposure, and after $\mathrm{Ar}^{+}$sputtering (Cu-sp.).
It is important to note, however, that the so-called WL cleaning effect is a very complex process that strongly depends on the detailed chemical structure of the contaminant layer and on the way it is bound to the underlying surface. In this, an important parameter can indeed be the time of irradiation, i.e., the dose given. Such an aspect has not been studied in detail since beam time was limited although initial investigations on the exposure dependence of the WL exposed sample indicated that irradiation for a few minutes and more than an hour produced similar spectra. This is consistent with the heavy dose given even after a few seconds of WL irradiation. Such doses have been shown to be sufficient to affect the desorption yield of the surface (hence its composition) [14,15]. It can be speculated that the WL exposure is, rather than cleaning the surface, changing it by cracking chemisorbed molecules to smaller units which can then no longer be photodesorbed. This is clearly supported by the striking difference seen in Fig. 5 between the spectrum for the WL irradiated sample and that obtained for clean copper: after WL illumination no Fermi level is observed, and the $\mathrm{Cu} 3 d$ bands have more the shape of the one observed in oxidized copper than of clean copper [13].

The shifts in the cutoff region observed confirms that WF changes will result from surface conditioning. In the case of sputtered $\mathrm{Cu}$ it exhibits a higher WF than the as-received sample and, therefore, is expected to have a lower PY. This result is consistent with the PY data which will be discussed in Secs. III D and IIIE.

\section{Photoemission with monochromatic light: $\mathrm{Cu}$-el.}

It is interesting to understand whether SR irradiation effects are comparable to the one obtained with annealing. To address this question an electropolished copper sample, $\mathrm{Cu}-\mathrm{el}$., was studied after different annealing treatments.

Figure 6 shows photoemission spectra taken at $50 \mathrm{eV}$ photon energy of such a $\mathrm{Cu}$ sample in three conditions: (a) as received, (b) after SR illumination $\left(10^{16}-10^{17}\right.$ photons $\left./ \mathrm{mm}^{2}\right)$, (c) after UHV annealing (pressure during annealing was less than $5 \times 10^{-9}$ mbar) at $(300 \pm 30){ }^{\circ} \mathrm{C}$ for $2.25 \mathrm{~h}$ (sample Cu-el.-an.).

The as-received material gives a very high emission of very low energy electrons (as in the case of $\mathrm{Cu}$ ), which is reduced in a similar way both after SR irradiation and after UHV annealing. However, the two surface treatments affect differently the surface electronic properties, as demonstrated by the strong differences observed in the $\mathrm{Cu} 3 d$ valence band structures. The annealing procedure seems to produce a cleaner surface than WL exposure, since the $\mathrm{Cu} 3 d$ valence band is more pronounced. This is in line with the observed higher activation energy of the molecules at a surface after exposure to SR than after annealing [15]. However, the annealing is less efficient than Ar sputtering in terms of cleaning the surface. 


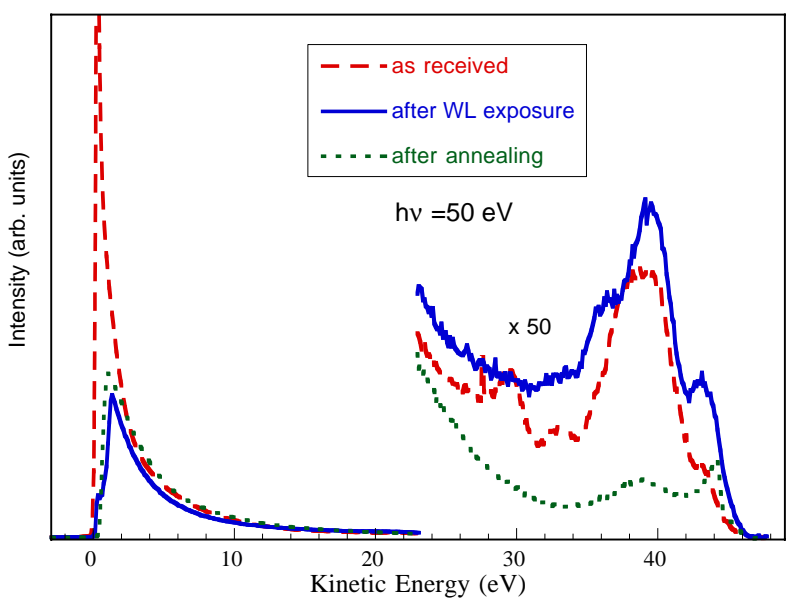

FIG. 6. (Color) The photoemission spectra, taken at $50 \mathrm{eV}$ photon energy, of $\mathrm{Cu}$-el. as received, after WL exposure, and after annealing in UHV (pressure during annealing was less than $\left.5 \times 10^{-9} \mathrm{mbar}\right)$ at $(300 \pm 50)^{\circ} \mathrm{C}$ for $2.25 \mathrm{~h}(\mathrm{Cu}$-el.-an. $)$.

\section{Photemission with monochromatic light: $\mathrm{TiZr}$}

A family of candidate materials for the LHC vacuum chambers are nonevaporable getters (NEGs) since they provide an elegant solution for distributed pumping in conductance limited vacuum systems [16]. In particular these materials are being proposed in the warm sections of the machine and in the experimental beam pipes. In order to pump, NEGs must be activated by heating. It is therefore of interest to study their behavior under SR illumination after activation.

A similar experiment to that described previously in Sec. IIIB 2 was performed on a $\sim 1 \mu \mathrm{m}$ TiZr film deposited onto OFE $\mathrm{Cu}$. The spectra are shown in Fig. 7.

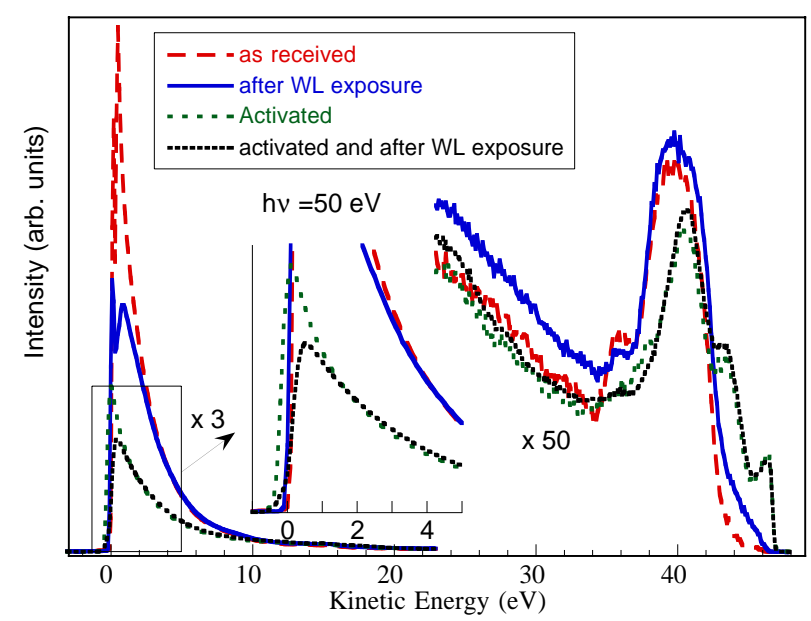

FIG. 7. (Color) The photoemission spectra, taken at $50 \mathrm{eV}$ photon energy, of TiZr as received, after WL exposure, after annealing in UHV (pressure during annealing was less than $10^{-8}$ mbar) at $(330 \pm 30)^{\circ} \mathrm{C}$ for $4 \mathrm{~h}$ (TiZr-ac.), and after $\mathrm{WL}$ exposure of the annealed surface.
The as-received surface shows a very high emission of low energy electrons.

Again, illumination with WL SR $\left(10^{16}-10^{17}\right.$ photons/ $\mathrm{mm}^{2}$ ) induces strong spectral changes: The secondary emission is reduced and structure closer to the Fermi edge in the top of the valence band appears. This is again a signature of the SR assisted cleaning.

More interestingly, the annealing of such a surface causes dramatic changes in its nature: The intensity of secondary electrons reduces and a clear Fermi level is observed as a signature of a clean metallic surface. The effect of annealing causes the removal of the oxide passivation layer present on the as-received surface. This sample was annealed in an UHV pressure of less than $1 \times 10^{-8}$ mbar and at $(330 \pm 30)^{\circ} \mathrm{C}$ for $4 \mathrm{~h}$. From these data the atomic cleanliness of the surface cannot be evaluated since valence band photoemission, for instance, is not sensitive to hydrogen, but the metallicity of the surface after activation, as shown from the appearance of a sharp Fermi edge at $46.8 \mathrm{eV}$ kinetic energy, is clearly demonstrated.

As in the case of $\mathrm{Cu}$-sp., the valence band structures are also unaffected by further WL illumination, perhaps suggesting that the surface is atomically clean. A closer examination of the secondary electron distribution, however, indicates that the WL modifies the distribution, both by affecting its intensity, and by shifting the zero kinetic energy cutoff. This shift is a signature of a related change in the WF of the material, possibly due to desorption of atomic species (like $\mathrm{H}$ ) which might be present on the surface even after activation. This behavior is not surprising given the fact that it is known that a variation of a very small quantity, in the submonolayers coverage range, on a surface [12] can induce dramatic changes in the WF (up to some eV in some cases). This aspect is an essential issue for LHC since the PY, one of the most important parameters in the simulations of the heat loads and electron cloud issues, is obviously strongly dependent on the WF of the chosen material.

To verify whether the behavior reported in Fig. 7 is dependent on the chosen annealing conditions, the experiment was repeated on a second TiZr sample, prepared in an identical fashion to the first. The TiZr II sample was annealed following a sightly different procedure than the TiZr. It was annealed in an UHV pressure of less than $5 \times 10^{-9}$ mbar and at $(300 \pm 30)^{\circ} \mathrm{C}$ for $2.25 \mathrm{~h}$.

The spectra reported in Fig. 8 show qualitative agreement with the spectra from the TiZr sample, discussed above. However, they clearly indicate that the detailed behavior of a surface is indeed strongly dependent on the fine details of its history and activation process.

The as-received spectrum from the TiZr II sample exhibited an even sharper secondary emission at low kinetic energies than that from the TiZr sample. This is due to the fact that the $50 \mathrm{eV}$ spectrum reported here was 


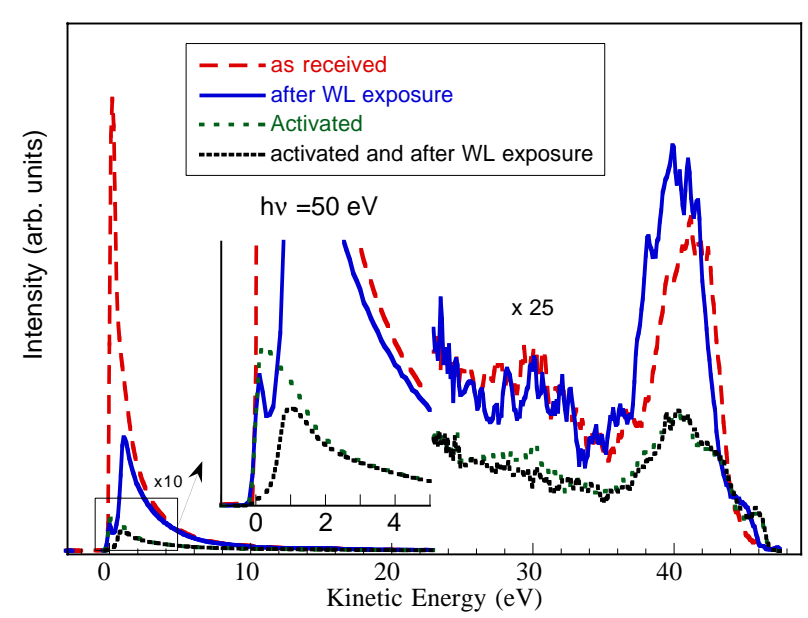

FIG. 8. (Color) The photoemission spectra, taken at $50 \mathrm{eV}$ photon energy, of TiZr II as received, after WL exposure, after annealing in UHV (pressure during annealing was less than $5 \times 10^{-9}$ mbar) at $(300 \pm 30)^{\circ} \mathrm{C}$ for $2.25 \mathrm{~h}$ (TiZr II-ac.), and after WL exposure of the annealed surface.

the first spectrum measured from the surface, whereas the corresponding spectrum from the TiZr sample was taken after some monochromatic light exposure performed at 20 , 30 , and $50 \mathrm{eV}$ photon energies (but before WL exposure).

The different annealing conditions result in observable changes in surface composition, and even if this second treatment exhibits a Fermi edge (indicative of essentially a metallic surface) the observed spectrum differs from the one reported in Fig. 7. It should be noticed that the second annealing process produces a surface which is also metallic but is much more sensitive to SR irradiation: The secondary electron distribution exhibits larger changes in intensity and energy position $(0.25 \mathrm{eV})$ reported in the inset of Fig. 8 than the one reported in Fig. 7.

These data clearly demonstrate the importance of defining a standard procedure to obtain reproducible surface conditions.

\section{Photoemission with monochromatic light: St 707}

A second type of nonevaporable getter material, namely St 707, has been studied. Figure 9 shows photoemission spectra taken at $50 \mathrm{eV}$ photon energy from a St 707 sample in three conditions: (a) as-received, (b) after WL illumination $\left(10^{16}-10^{17}\right.$ photons $\left./ \mathrm{mm}^{2}\right)$, (c) after UHV annealing (pressure during annealing was less than $5 \times$ $10^{-9}$ mbar at $(300 \pm 30){ }^{\circ} \mathrm{C}$ for $8 \mathrm{~h}$.

Once again, the as-received material exhibits a very high emission of very low energy electrons while the valence band signal is very weak and does not show any signal at the Fermi level.

Illumination with a WL dose of about $10^{16}$ $10^{17}$ photons $/ \mathrm{mm}^{2}$ induces quite dramatic changes to the surface. Not only does the secondary tail decrease significantly while the high energy electron emission increases

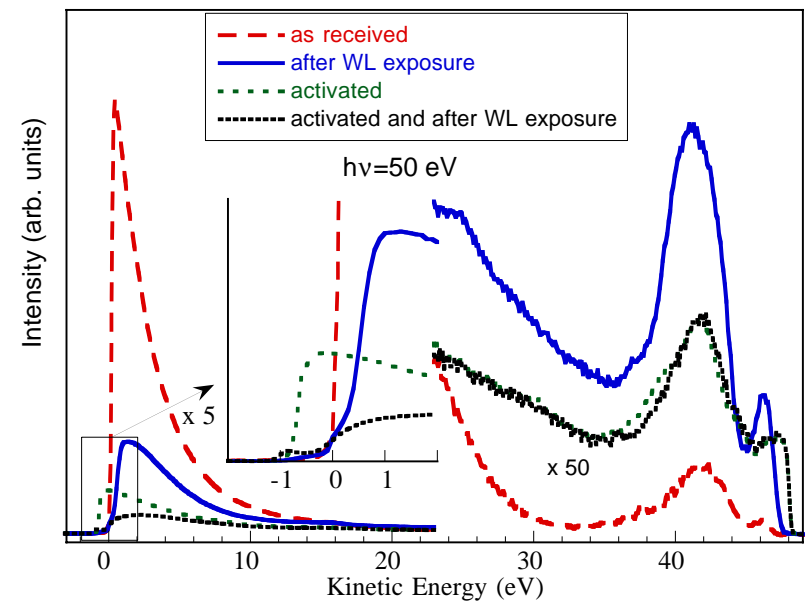

FIG. 9. (Color) The photoemission spectra, taken at $50 \mathrm{eV}$ photon energy, of Si 707 as received, after WL exposure, after annealing in UHV (pressure during annealing was less than $5 \times 10^{-9}$ mbar $)$ at $(300 \pm 30){ }^{\circ} \mathrm{C}$ for $8 \mathrm{~h}(\mathrm{St} 707$-ac. $)$, and after WL exposure of the annealed surface.

by more than a factor of 5 , but no line shape changes are observed.

As expected, annealing changes very significantly the surface from the appearance of a well defined Fermi level at $46.1 \mathrm{eV}$ implying that activating the St 707 getter produces a metallic surface. This spectrum is very different from the one obtained just after WL irradiation, indicating that the WL exposure is not as efficient in cleaning the surface. Together with these changes in the high energy part of the spectrum a significant intensity reduction and a large energy shift in the low energy region is observed.

Subsequent irradiation of the activated getter with WL does not affect significantly the valence band while, as in the case of TiZr, the secondary tail shifts back to higher energy with a reduction in intensity.

In this case there is evidence that WL irradiation induces additional modifications of the activated surface, observed by the appearance of the double low energy cutoff after WL reexposure. This is most probably due to the coexistence of differently cleaned regions during activation. This additional modification induced by WL may be due to removal of "light" contaminants from the surface which do not have consequences on the valence band line shape, but only in the cutoff region.

\section{Photoemission with monochromatic light: Pd}

Another interesting sample studied was Pd due to its affinity to $\mathrm{H}_{2}$, potentially increasing the pump speed of a NEG, once heated to about $150{ }^{\circ} \mathrm{C}$ [17]. In Fig. 10, the selected spectra obtained from such a sample according to different preparation procedures are shown.

This is the only case where WL exposure, with a dose of around $10^{16}-10^{17}$ photons $/ \mathrm{mm}^{2}$, does not change 


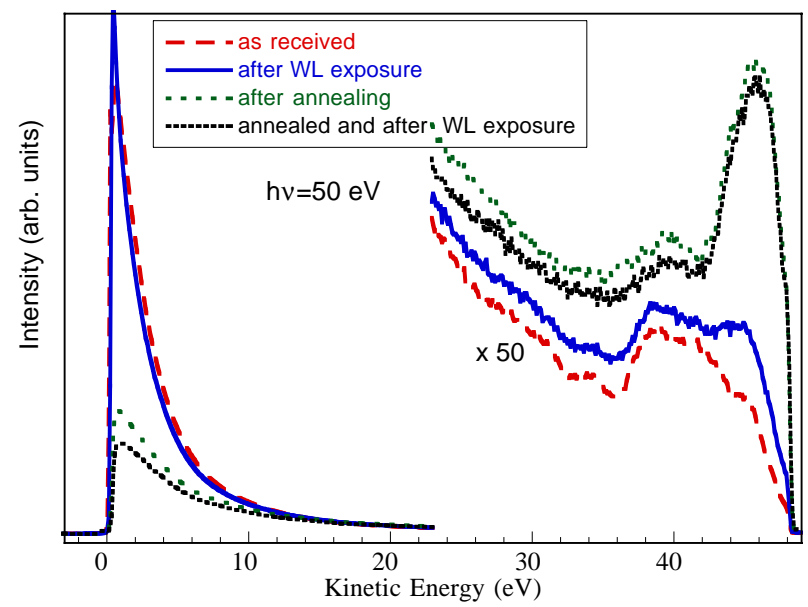

FIG. 10. (Color) The photoemission spectra, taken at $50 \mathrm{eV}$ photon energy, of $\mathrm{Pd}$ as received, after WL exposure, after annealing in UHV (pressure during annealing was less than $5 \times 10^{-9}$ mbar) at $(300 \pm 30)^{\circ} \mathrm{C}$ for $8 \mathrm{~h}$ (Pd-an.), and after WL exposure of the annealed surface.

significantly the spectral shape both at low energy and in the valence band region. Only annealing affects the electron energy distributions, once again decreasing the secondary electron tail intensity and increasing the emission in the valence band region. The valence band region of the annealed sample, both before and after WL exposure, shows a clear Fermi edge cutoff at $46.5 \mathrm{eV}$, the signature of an "almost clean" metallic surface.

\section{Photoemission with monochromatic light: TiN}

The PEP-II low energy (positron) ring will operate at nominal energy of $3.1 \mathrm{GeV}$ and current of $2.1 \mathrm{~A}$ [11]. A TiN coating has been chosen for this entire ring in order to eliminate the predicted electron cloud induced beam instabilities otherwise foreseen in that machine. For this reason experiments were performed on a TiN coated $\mathrm{Al}$ sample [11] which appeared black, carbonlike, and not Au-like characteristic of TiN. The sample was studied as-received, after $\mathrm{Ar}^{+}$sputtering at $2 \mathrm{keV}$ for $5 \mathrm{~min}$ in 1.3 mbar of $\mathrm{Ar}$ (estimated dose $2 \times 10^{16} \mathrm{Ar} \mathrm{cm}^{-2}$ ), and after dosing it with WL $\left(10^{16}-10^{17}\right.$ photons $\left./ \mathrm{mm}^{2}\right)$.

The WL effect on the as-received sample was not studied and therefore we can only speculate that this sample will also behave in a similar way as the others studied.

The sputtering was used to obtain a "cleaner surface." The measured spectra, reported in Fig. 11, show that the sputtering process changes the weight of the emitted electrons from the valence band to the low energy part of the spectrum. This effect is contrary to any of the cleaning procedures used on the other samples analyzed, perhaps requiring further investigations. However, it should be noted that the sample contains $43 \%$ oxygen and $22 \%$ carbon in the bulk [18] and therefore removal of the outermost layers

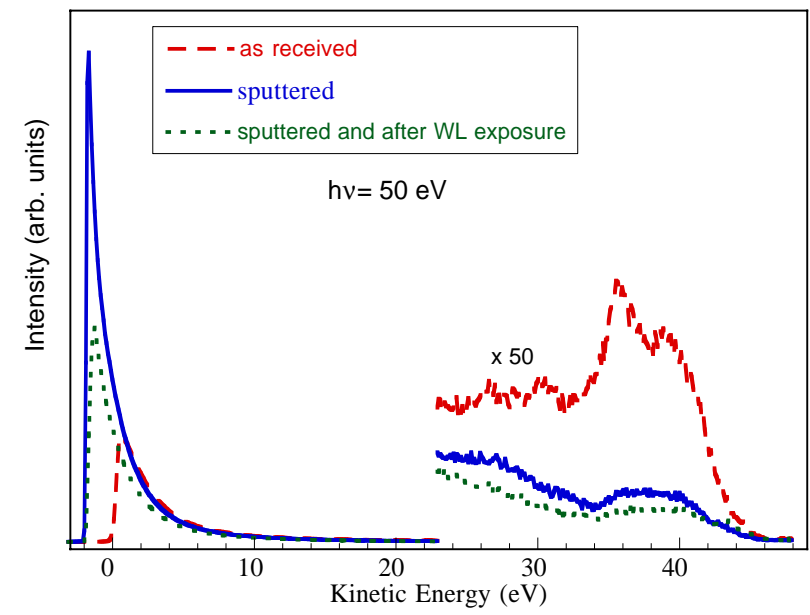

FIG. 11. (Color) The photoemission spectra, taken at $50 \mathrm{eV}$ photon energy, of TiN as received, after $\mathrm{Ar}^{+}$sputtering (TiNsp.), and after WL exposure of the sputtered surface.

by sputtering will not necessarily result in the exposure of a cleaner surface. Not only the sputtering process affects the intensity of the electron distribution, but also the cutoff energy, showing that the cleaning procedure reduces the WF of the surface by more than $2 \mathrm{eV}$.

\section{The photon energy dependence of the PY (constant final state spectroscopy)}

In the arcs of the LHC the $44.1 \mathrm{eV}$ critical energy SR, with a beam divergence of $0.55 \mathrm{mrad}$ for $5 \mathrm{eV}$ photons, will illuminate the beam screen at a mean incidence angle of $4.5 \mathrm{mrad}$. Specular photon reflection from perfectly smooth surfaces, at such energies and incidence angles, might be expected to be close to $100 \%$ [8]. Such calculations, at a given incidence angle, predict that the low energy photons (VUV) are reflected more readily than the higher energy photons (soft $x$ ray). Real surfaces with a finite roughness are far from being perfect reflectors in the VUV and a certain percentage of the incident photons will be adsorbed and/or diffused. Photons in the UV energy range can be reflected significantly from polished surfaces even when impinging at near normal incidence [19]. One may speculate therefore that a significant intensity of diffusely scattered UV light exists from a real surface. Experiments using monochromatic light will be required to disentangle this question of photon reflection to produce a realistic estimate of the intensity and spectral distribution of the reflected and/or diffusely scattered photons. Nevertheless, it is expected that the spectrum of reflected and/or diffused WL will not resemble the one shown in Fig. 2, but will be more weighted toward the low energy. This therefore leads to the importance of analyzing the total yield of the different materials as a function of photon energy. In the case of a material which exhibits a very low cross section for photons with energies 
less than, say, $30 \mathrm{eV}$, multiply reflected/diffused light may be neglected. On the other hand, multiply reflected/ diffused photons will be an important consideration for a material exhibiting a high cross section for such low energy photons.

The availability of a tunable monochromatic light provides the possibility to study not only the energy distribution curves as a function of photon energy, as it was presented in Sec. III A, but also the PY as a function of photon energy. The measurement was made by recording the number of secondary electrons emitted at $1.0 \pm$ $0.2 \mathrm{eV}$ kinetic energy while scanning the photon energy from 18 to $110 \mathrm{eV}$. This so-called CFS spectroscopy is well known to mimic the shape of the total yield (i.e., drain current) emitted per monochromatic incident photon [13]. The total PY could not, unfortunately, be measured directly for technical reasons.

In Fig. 12, CFS for four materials, namely, $\mathrm{Au}, \mathrm{Cu}-\mathrm{el}$., TiZr-an., and Al, are shown. It is evident that the four curves are different reflecting the different cross sections of the atomic elements. While $\mathrm{Au}$ and $\mathrm{Al}$ are such that the maximum electron emission is caused by photons in the energy range between 30 and $100 \mathrm{eV}$, electrons from $\mathrm{Cu}$-el. and TiZr-an. are mainly caused by photons of 20 to $40 \mathrm{eV}$ and 30 to $50 \mathrm{eV}$, respectively. It is not possible here to quantify the implication of the available data for the LHC; nevertheless, this preliminary study points to the need of a better understanding of the photon energy dependence of the emitted photoelectrons and of the specularly reflected and/or diffusely reflected light.

\section{White light photoelectron yields of the as-received samples}

One of the important parameters relevant to the existence of a potentially serious electron cloud in the LHC is the PY per adsorbed photon. The measured drain currents and deduced PY of the as-received samples are indicated in Table III. Also shown is the Au reference whose PY is in excellent agreement with literature [9]. It should be noted that the reported PY is that for the number of emitted electrons per incident photon and not, as reported in other works, per adsorbed photon [20]. The error in the PY, given in Table III, is estimated here only from the error in the photon flux (see Sec. II B for details). In Fig. 13 these data are plotted as a function of decreasing PY. The plotted error bars are not the errors quoted in Table III (errors in determining the absolute flux) but are the reproducibility errors found from measuring two different TiZr samples (TZr and TiZr II) and two different $\mathrm{Cu}$-el. samples (the results from the second sample are not shown). There the PYs were in agreement to within \pm 0.002 . In other words, the absolute PYs are accurate to within \pm 0.01 , whereas the relative PYs are accurate to within \pm 0.002 .

Table III and Fig. 13 show that the WL PY from the as-received surfaces exhibit values in the range $(0.053-$ $0.106) \pm 0.010$. The Al gives the highest PY twice that of the St 707. The fact that most of the PY appear not to differ substantially implies that the surface properties of these materials after the WL irradiation are similar. An
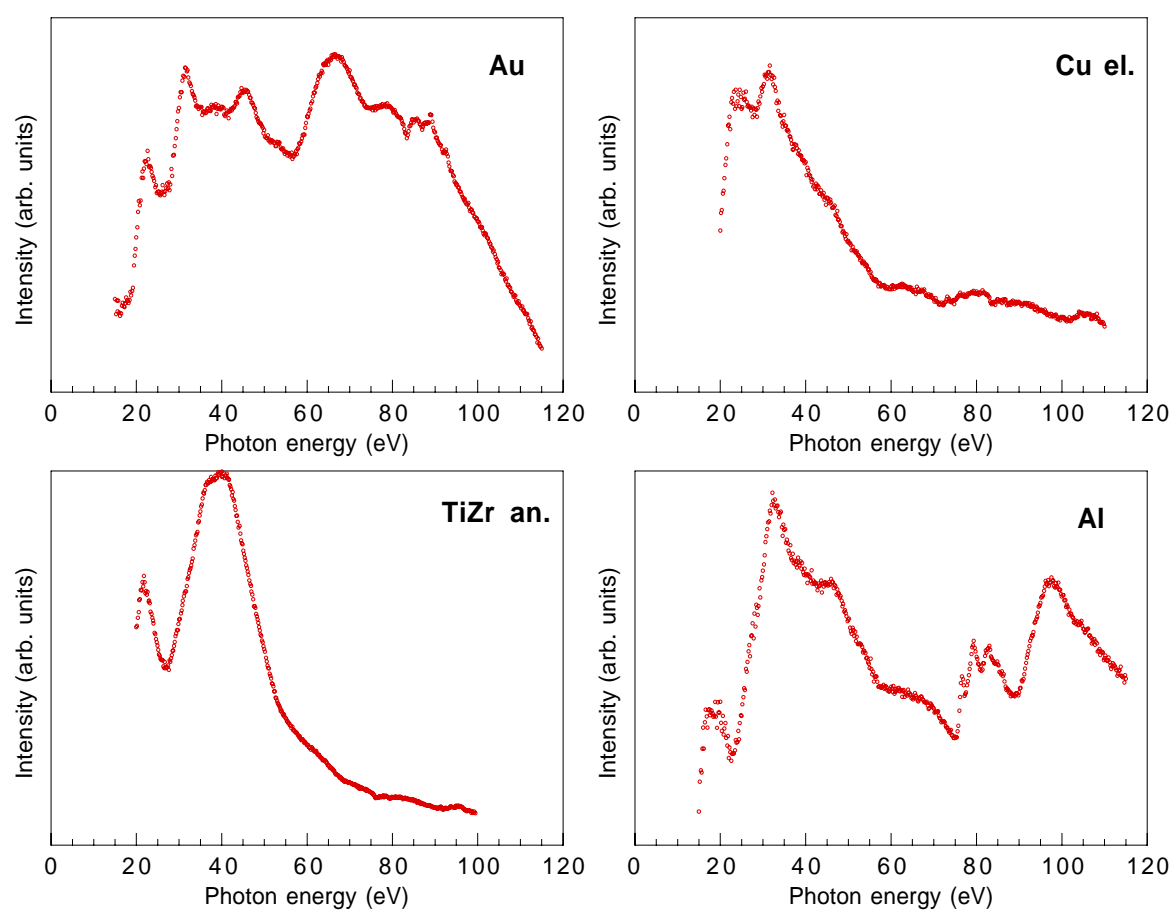

FIG. 12. (Color) The CFS spectra from Au, Cu-el., TiZr-an., and Al in the photon energy range from 18 to $110 \mathrm{eV}$. The collected electrons are emitted at $1.0 \pm 0.2 \mathrm{eV}$ kinetic energy. 
TABLE III. The PY per incident photon for the different asreceived samples studied. In the second and third columns the sample drain currents and the last refocusing mirror drain currents are reported, respectively. In the fourth column the estimated photon flux is given (see Sec. II A) for details. In the last column the PY is reported with its absolute error.

\begin{tabular}{cllcc}
\hline \hline Sample & $\begin{array}{c}I_{\text {sample }}(\mu \mathrm{A}) \\
(\mu \mathrm{A})\end{array}$ & $\begin{array}{c}I_{\text {mirror }} \\
\left(\mu \text { photons } /\left(\mathrm{s} \mathrm{mm}^{2}\right)\right]\end{array}$ & $\begin{array}{c}\text { Photon flux } \\
\text { (electrons/photon) }\end{array}$ \\
\hline $\mathrm{Au}$ & 3.72 & 3.59 & $5.6 \times 10^{14}$ & $0.041 \pm 0.010$ \\
$\mathrm{Cu}$ & 3.8 & 2.4 & $3.8 \times 10^{14}$ & $0.063 \pm 0.010$ \\
$\mathrm{Cu}-\mathrm{ab}$. & 1.94 & 0.83 & $1.5 \times 10^{14}$ & $0.093 \pm 0.010$ \\
$\mathrm{TiN}$ & 8.3 & 4.2 & $6.5 \times 10^{14}$ & $0.080 \pm 0.010$ \\
$\mathrm{TiZr}$ & 2.4 & 1.1 & $1.7 \times 10^{14}$ & $0.088 \pm 0.010$ \\
$\mathrm{TiZr} \mathrm{II}$ & 1.6 & 0.76 & $1.2 \times 10^{14}$ & $0.084 \pm 0.010$ \\
$\mathrm{Cu}-\mathrm{el}$. & 1.5 & 0.88 & $1.4 \times 10^{14}$ & $0.070 \pm 0.010$ \\
$\mathrm{Pd}$ & 0.87 & 0.48 & $7.5 \times 10^{13}$ & $0.072 \pm 0.010$ \\
$\mathrm{St} \mathrm{707}$ & 4.9 & 3.6 & $5.7 \times 10^{14}$ & $0.053 \pm 0.010$ \\
$\mathrm{Al}$ & 9.0 & 3.45 & $5.4 \times 10^{14}$ & $0.106 \pm 0.010$ \\
\hline \hline
\end{tabular}

explanation for this result may be that photocracking of similar molecules, found on all the as-received samples, produces similar fragments left behind on the surface after the intense WL SR. This surface layer will therefore smooth out any expected differences in the PY of the differential materials.

It should be pointed out that the photon flux used to obtain these data was approximately 1000 times more intense than that expected in the LHC, therefore these reported PYs are not representative of the initial PY to be expected in the LHC, rather they are those to be expected after a considerable time of machine operation (estimated to be 5 to $50 \mathrm{~h}$ of LHC nominal operation). This aspect will be addressed in Sec. III G, where the reconstruction of low dose white light spectra is presented.

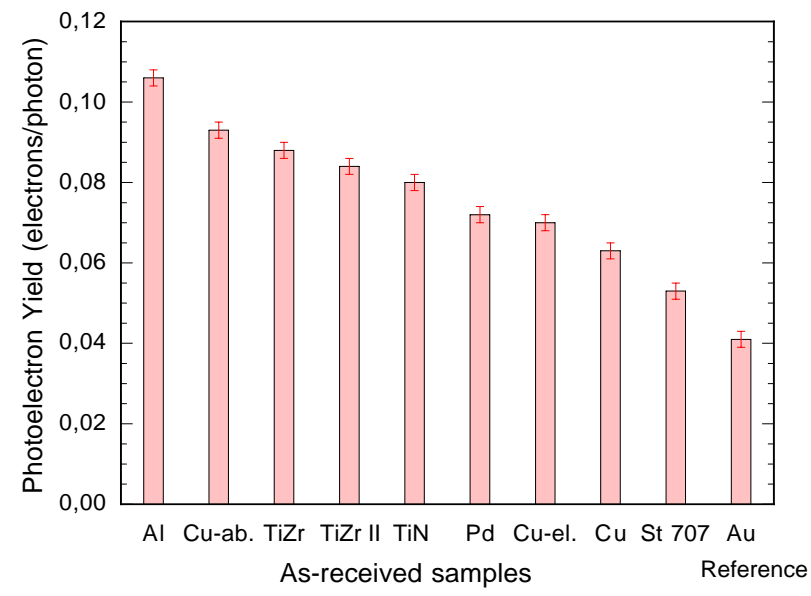

FIG. 13. (Color) The plot showing the PY during WL illumination, sorted as a function of decreasing values, for the different as-received samples.

\section{E. White light photoelectron yields of the surface conditioned samples}

For comparison, Fig. 14 shows the measured PY of the as-received and conditioned samples. The most dramatic change on surface conditioning is seen for the $\mathrm{Al}$ sample where the PY increases by almost a factor of 8 after sputtering. Sputtering of the TiN samples has a similar, but less marked, enhancement $(50 \%)$ on its PY. This could be well explained by the decrease of WF observed in Fig. 11. On the other hand, sputtering of the $\mathrm{Cu}$ sample causes a marginal reduction (16\%) on its PY. As shown in Sec. III B, sputtering can clean surfaces very efficiently by removing the outermost layers of the sample, resulting in the underlying material to become more exposed. The large variations between the PY of the WL exposed surfaces and the conditioned surfaces leads to the notion that the surfaces exposed to WL are effectively "passivated" by their oxide layer.

Activation of TiZr, TiZr II, or St 707 results in a reduced PY by between $38 \%$ and 32\%, respectively. However, annealing of either the $\mathrm{Pd}$ or $\mathrm{Cu}$-el. samples changes little the PY. The effect of activation of such NEGs to $300{ }^{\circ} \mathrm{C}$ partially removes the outermost surface oxide layers and therefore exposes the substrate material [21]. The applied annealing of the $\mathrm{Pd}$ and $\mathrm{Cu}$-el. does not activate such surfaces, being insufficient to modify the outermost surface oxide layers and hence their PY.

The lowest PY of 0.035 was measured for the activated St 707 surface, being slightly lower than that of the evaporated Au reference surface.

\section{F. Energy distributions of the WL EDC}

In addition to the PY presented in Secs. IIID and III E, the energy distribution of the PY is relevant to the LHC electron cloud phenomena (multipacting, induced gas desorption, heat loads, etc.). Since the estimated heat loads and electron cloud growth are expected to be sensitive

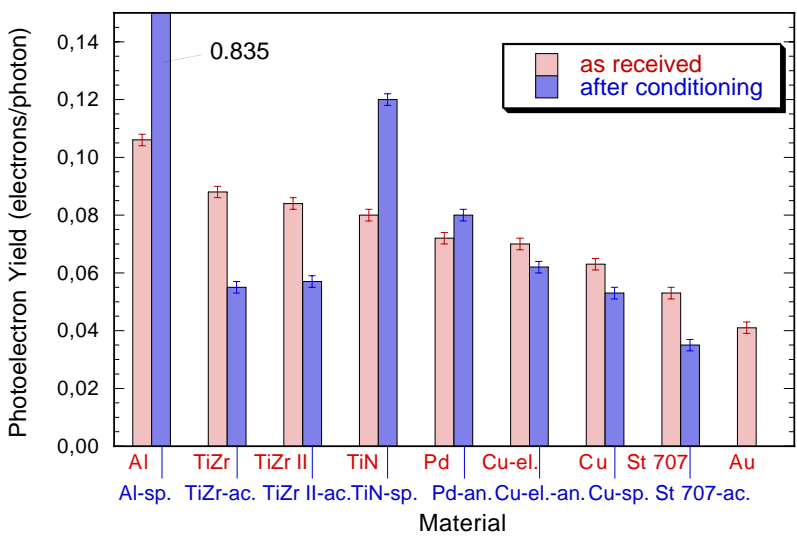

FIG. 14. (Color) The WL PY for the samples in their asreceived and conditioned states. 


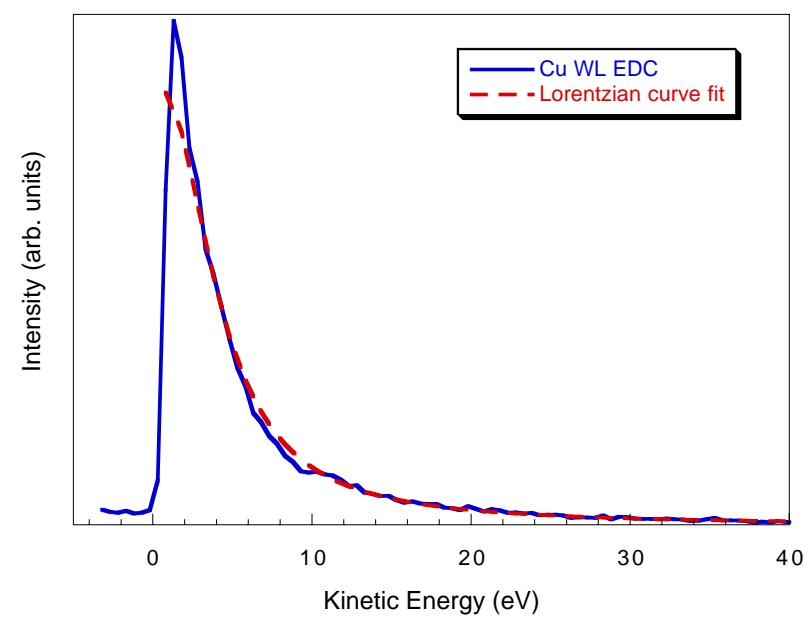

FIG. 15. (Color) The Lorentzian fit to $\mathrm{Cu}$ WL EDC.

to the energy distribution of the emitted electrons [3,4], realistic line shapes should be included in the simulations. As an example, the WL EDC for the $\mathrm{Cu}$ sample is shown in Fig. 15. The most appropriate description of the energy distribution in this case was found to be Lorentzian in nature centered at $0.64 \mathrm{eV}$ and width $\sigma$ of $3.7 \mathrm{eV}$. It is shown in the following that it is not possible to describe the photoelectron distributions of different materials with similar Lorentzians scaled to their PY intensities. In each separate case experimental input is required to obtain the realistic electron distribution to be used in the simulations.

In Fig. 16 are shown the different WL excited energy distribution spectra for the different as-received samples studied (solid line). There it is clear that the electron energy distributions are significantly different for the different samples, in contrast with the similar PY reported in Secs. III D and III E and recalled in the insets of Fig. 16. These WL exposed spectra clearly demonstrate that it is not only sufficient to measure the PY, but it is also important to measure the energy distribution of the emitted electrons. As an example, the $\mathrm{Pd}$ spectrum and the $\mathrm{Cu}$-el. spectrum give nearly identical PYs $(0.072$ and 0.070 , respectively), but their energy distributions are considerably different; the former exhibits a sharp distribution of photoelectrons with mainly very low kinetic energies, and in the latter, together with a sharp emission of very low kinetic energy electrons, a broader peak is observed.

The WL EDC for the conditioned samples (dashed curves in Fig. 16) demonstrate that significant changes in the line shapes occur due to the conditioning. As an example, the WL exposed Cu-el. sample exhibits a sharp narrow peak at low energy and a broader peak, centered at $2 \mathrm{eV}$ kinetic energy, with a characteristic tail to higher kinetic energies. After annealing the peak at $2 \mathrm{eV}$ is shifted by $3 \mathrm{eV}$ to higher energy and is considerably broadened.

\section{G. Generation of low dose WL spectra}

At this point it is important to recall that the monochromatic spectra, presented in Sec. III B, suggest strongly that the WL spectra of the as-received and conditioned samples, presented in the previous sections, were subject to modifications due to the significant irradiation dose of WL during their acquisition $\left(10^{16}-10^{17}\right.$ photons $/ \mathrm{mm}^{2}$
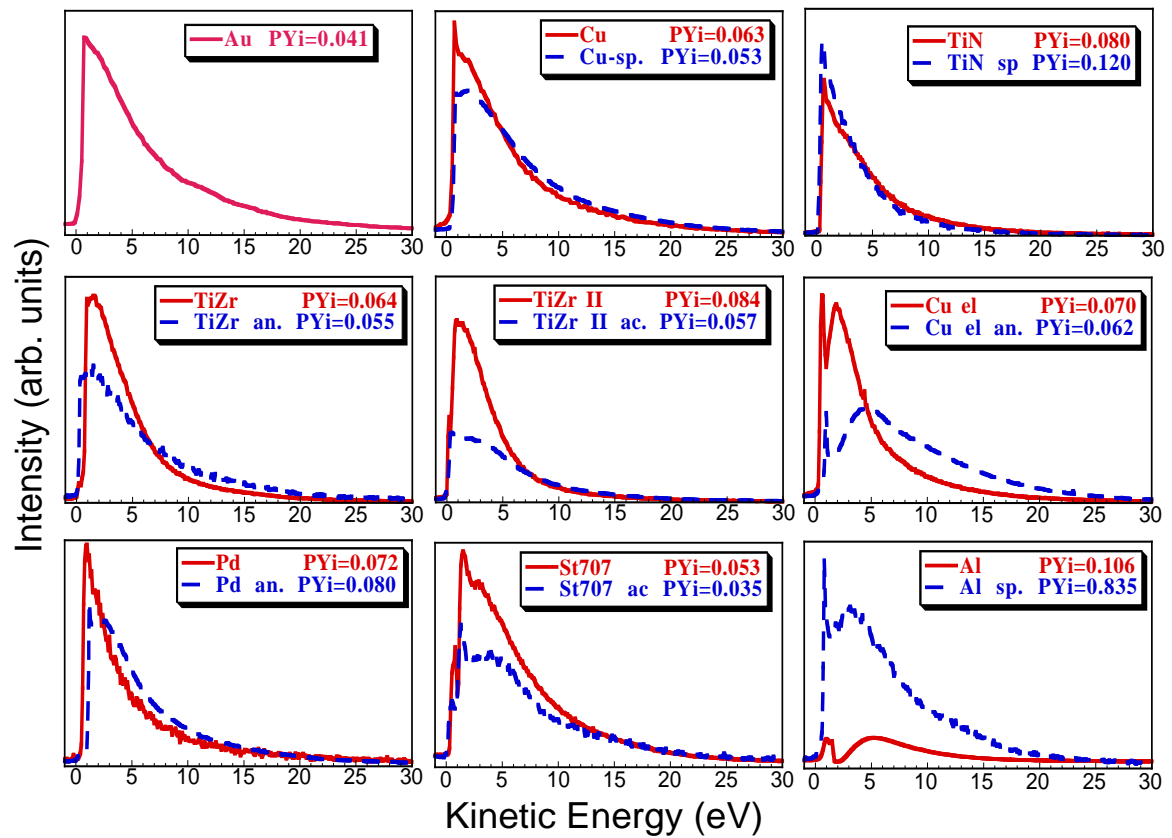

FIG. 16. (Color) The WL excited EDCs. The spectra from as-received (conditioned) samples are depicted with red solid (blue dashed) lines. 
corresponding to 5 to $50 \mathrm{~h}$ of LHC nominal operation). In order to provide information on the initial PY expected in the LHC, i.e., before any significant SR radiation cleaning has occurred, a WL EDC can be reconstructed from a summation of the acquired monochromatic spectra, hereafter called a "low photon dose" WL spectrum; such a photon dose corresponds to less than $1 \mathrm{~min}$ of LHC nominal operation.

Such a spectrum, however, may be inaccurate due to the limited number of monochromated spectra from which it is generated. The analysis of the evaporated $\mathrm{Au}$ sample serves to test the validity procedure since clean Au should not exhibit any difference if the electron distribution has been measured before or after high photon dose irradiation. The resulting differences in the two Au spectra reported in Fig. 17 therefore derive the inaccuracy in the monochromatized spectra summation. The observed agreement is quite good (the integral of the two curves differ by less than $13 \%$ ) giving confidence to the procedure. These data allow us to determine the
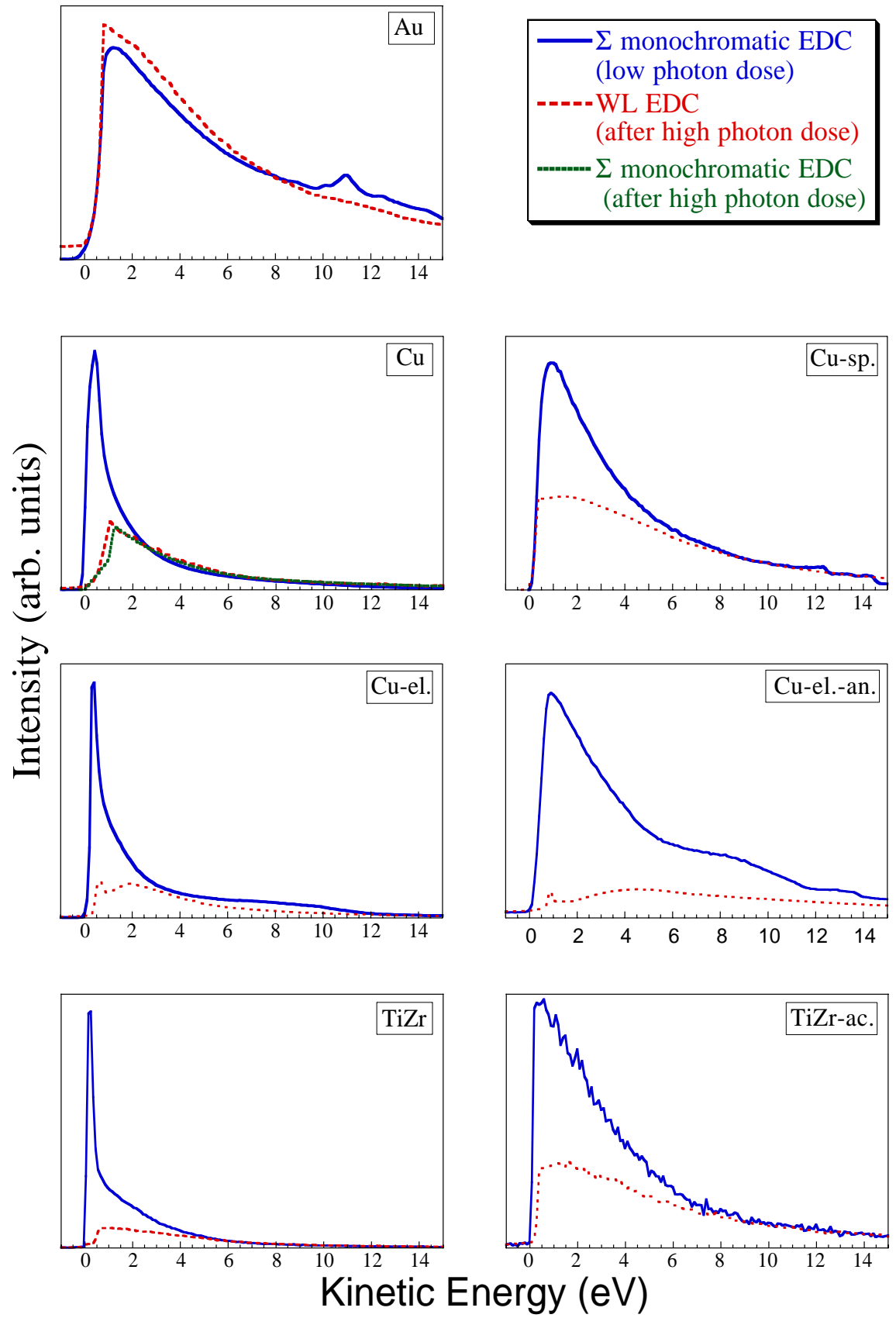

FIG. 17. (Color) Selected low photon dose spectra generated from a summation of the monochromated EDCs (blue solid line) compared with their corresponding WL EDC (red dashed line). The sum of the monochromatic EDCs (after high photon dose) is only shown for $\mathrm{Cu}$ (green xxx line). 
absolute error bar on the value of the PY to be around 0.02 or less. In addition, lending further credence to the procedure, the reconstructed $\mathrm{Cu}$ spectrum from after a high photon dose, i.e., after WL illumination, is very similar to its corresponding WL EDC as can be seen in Fig. 17 (second plot from the top, left panel).

In Fig. 17 a selection of a low photon dose WL spectra is shown in comparison with its corresponding WL EDC from Fig. 16. Here the low photon dose WL spectrum has been normalized to the high energy tail of the WL EDC. It can be seen that significant differences between the two spectra exist. In the case of the as-received $\mathrm{Cu}$ and TiZr the low photon dose WL spectrum is dominated by a narrow and intense peak $(<1 \mathrm{eV} \mathrm{FWHM})$ at low kinetic energies which is almost totally absent in the latter (a small remnant structure is observed). Even once activated the TiZr low photon dose WL spectrum has significantly more intensity below about $6 \mathrm{eV}$ kinetic energy than the WL spectrum. These intensity and line shape modifications can be attributed directly to sample cleaning by SR.

Figures 18 and 19 show that most materials exhibit a more intense low photon dose WL PY than that measured with WL. The most striking differences are seen for the as-received samples, exhibiting an intense narrow peak at low kinetic energy that is suppressed once irradiated with high dose WL. The air-baked $\mathrm{Cu}$ sample exhibits the strongest reduction in PY, by a factor of 5, whereas St 707 shows no significant modification; however, its EDCs are significantly different (not shown). The data shown in Fig. 19, for the conditioned samples, indicate that WL induces additional modifications to the initial PY, reducing significantly the PY of the TiNsp. and Cu-el.-an. surfaces. Indeed, in Secs. III B 6 and III 2 these surfaces were found not to be clean after conditioning.

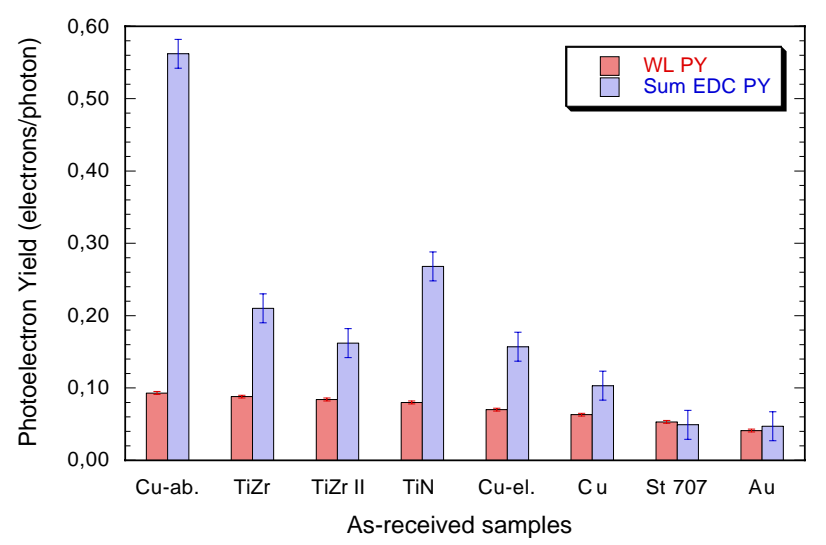

FIG. 18. (Color) A comparison between WL PY and its corresponding low photon dose WL PY generated from a summation of the acquired EDCs for the as-received samples. Error bars are those generated from the Au reconstruction.

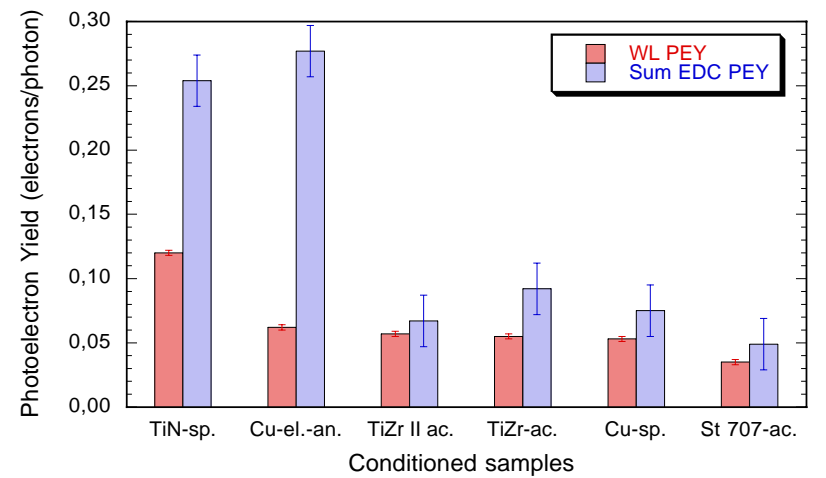

FIG. 19. (Color) A comparison between WL PY and its corresponding low photon dose WL PY generated from a summation of the acquired EDCs for the conditioned samples. Error bars are those generated from the $\mathrm{Au}$ reconstruction.

\section{H. The energy distribution of the secondary electrons in the $\mathrm{LHC}$}

The results discussed in the previous paragraphs have consequences if applied to the analysis of the SY. This process, subsequent to the photoelectron emission, contributes to the electron cloud instability buildup and has been carefully addressed in recent theoretical studies $[3,4]$. An electron created by photoemission will be accelerated, to a mean energy of $380 \mathrm{eV} \mathrm{[3]} \mathrm{towards} \mathrm{the}$ opposite wall of the beam screen, due to the contemporary presence of the proton beam. This electron will then create a cascade of secondaries that may be further accelerated against the opposite walls by following bunches. The study of the energetics and the time structure of the multipacting problem suggest that only secondary electrons with kinetic energy between 1 and $6 \mathrm{eV}$ may play a role in the buildup of the electron cloud instability $[3,4]$.

Secondary electrons are emitted from a surface by impinging photons or electrons. Their origin is due to the multiple scattering of a photoexcited electron or of the incident electron within the solid. This multiple scattering causes a cascade of electrons towards lower energy, socalled secondary electrons. These analogous processes of secondary electrons creation suggest that they will result in secondary electrons with similar energy distributions. However, their intensities will be quite different due to the longer mean free path of the photon to that of the electron. Despite this assumption, requiring careful experimental verification, the present data can be used to extract information for the LHC electron cloud phenomenon.

Taking an integral curve of the EDCs, scaled to the PY, one can obtain a relative PY that is proportional to the SY in a defined energy window. Table IV shows a compilation of the calculated yields in the energy window 1 to $6 \mathrm{eV}$ together with the total yield. From this table, with the assumption that the PY is proportional to the $\mathrm{SY}$, it is evident that if one should judge the annealing 
TABLE IV. The low dose WL total yield, reconstructed from a summation of the EDCs, high photon dose WL total yield measured with WL, and corresponding WL yield in the energy window, 1-6 eV, for the different samples studied.

\begin{tabular}{ccccc}
\hline \hline & $\begin{array}{c}\text { Low dose } \\
\text { WL yield } \\
\text { (electrons/photon) }\end{array}$ & $\begin{array}{c}\text { Energy window } \\
\text { low dose } \\
\text { WL yield } \\
\text { (electrons/photon) }\end{array}$ & $\begin{array}{c}\text { WL yield } \\
\text { (electrons/photon) }\end{array}$ & $\begin{array}{c}\text { Energy window } \\
\text { WL yield } \\
\text { (electrons/photon) }\end{array}$ \\
\hline Sample & $0.047 \pm 0.020$ & $0.022 \pm 0.020$ & $0.041 \pm 0.002$ & $0.023 \pm 0.002$ \\
$\mathrm{Au}$ & $0.103 \pm 0.020$ & $0.051 \pm 0.020$ & $0.063 \pm 0.002$ & $0.042 \pm 0.002$ \\
$\mathrm{Cu}-$ sp. & $0.075 \pm 0.020$ & $0.046 \pm 0.020$ & $0.053 \pm 0.002$ & $0.026 \pm 0.002$ \\
$\mathrm{Cu}-\mathrm{ab}$. & $0.562 \pm 0.020$ & $0.290 \pm 0.020$ & $0.093 \pm 0.002$ & $0.058 \pm 0.002$ \\
TiN & $0.268 \pm 0.020$ & $0.125 \pm 0.020$ & $0.080 \pm 0.002$ & $0.044 \pm 0.002$ \\
TiN-sp. & $0.254 \pm 0.020$ & $0.132 \pm 0.020$ & $0.120 \pm 0.002$ & $0.075 \pm 0.002$ \\
TiZr & $0.210 \pm 0.020$ & $0.102 \pm 0.020$ & $0.088 \pm 0.002$ & $0.055 \pm 0.002$ \\
TiZr-ac. & $0.092 \pm 0.020$ & $0.055 \pm 0.020$ & $0.055 \pm 0.002$ & $0.027 \pm 0.002$ \\
TiZr II & $0.162 \pm 0.020$ & $0.073 \pm 0.020$ & $0.084 \pm 0.002$ & $0.053 \pm 0.002$ \\
TiZr II ac. & $0.067 \pm 0.020$ & $0.037 \pm 0.020$ & $0.057 \pm 0.002$ & $0.027 \pm 0.002$ \\
Cu-el. & $0.157 \pm 0.020$ & $0.069 \pm 0.020$ & $0.070 \pm 0.002$ & $0.042 \pm 0.002$ \\
Cu-el.-an. & $0.277 \pm 0.020$ & $0.146 \pm 0.020$ & $0.062 \pm 0.002$ & $0.021 \pm 0.002$ \\
Pd & $\ldots$ & $\ldots$ & $0.072 \pm 0.002$ & $0.043 \pm 0.002$ \\
Pd-an. & & $\ldots$ & $0.080 \pm 0.002$ & $0.049 \pm 0.002$ \\
St 707 & $0.049 \pm 0.020$ & $0.026 \pm 0.020$ & $0.053 \pm 0.002$ & $0.029 \pm 0.002$ \\
St 707-ac. & $0.049 \pm 0.020$ & $0.017 \pm 0.020$ & $0.035 \pm 0.002$ & $0.020 \pm 0.002$ \\
Al & $\ldots$ & $\cdots$ & $0.106 \pm 0.002$ & $0.038 \pm 0.002$ \\
Al-sp. & $\ldots$ & $\ldots$ & $0.835 \pm 0.002$ & $0.362 \pm 0.002$ \\
\hline \hline
\end{tabular}

treatment as a process to change the heat load only from the total yield measured we should conclude, for example, that annealing of the $\mathrm{Cu}$-el. sample will decrease it only by about $10 \%$ (assuming that the heat load scales linearly with the number of emitted electrons). The possibility of computing the number of electrons emitted in the energy window shows that annealing the $\mathrm{Cu}$-el. reduces the heat load by about 50\% which is a significantly different conclusion than that drawn from the total yields. However, this conclusion cannot be extrapolated to other materials. Indeed, if in the case of TiZr we measure about $25 \%$ reduction in total yield it corresponds to about 50\% reduction in the electron emission in the 1 to $6 \mathrm{eV}$ energy window. In the case of $\mathrm{Pd}$, annealing causes a $10 \%$ increase both in the yield and in the number of electrons emitted in the $1-6 \mathrm{eV}$ energy window.

It is clear that more studies are required both in the case of WL illumination EDC and electron bombardment induced EDC. Here we demonstrate the importance of studying the energy distribution of the photoemitted electrons and speculate that a similar experimental approach should be necessary to understand the acceptable performance of LHC candidate materials in terms of their electron induced production of secondaries.

\section{CONCLUSIONS}

SR photoemission has been shown to be a powerful tool to study surface properties of relevance for the LHC vacuum chamber optimization. It has been shown how the energy and angular distribution of the emitted photoelectrons can be measured. Irradiation of samples with SR has shown that the total PY is mainly due to low energy electrons.

The EDCs of such as-received and conditioned samples demonstrated that a universal curve describing their shape does not exist. The secondary electron distribution of the as-received $\mathrm{WL}$ irradiated $\mathrm{Cu}$ sample was found to be Lorentzian in nature. WL EDCs of samples subjected to a low photon dose were reconstructed from monochromatic spectra. As-received samples exhibit an intense and narrow secondary electron peak whereas surface conditioned samples exhibit a broader secondary distribution. The implications on the LHC cryogenic heat load of such different EDCs shapes are currently analyzed through simulations.

Most of the samples subjected to a low photon dose exhibit a higher PY than those subjected to a high dose. Intense WL irradiation of as-received samples results in a similar PY for all the studied samples; Al gives the highest PY (0.106) while Au give the lowest (0.041). An explanation that the WL exposed PY do not differ greatly between most of the samples may be the result of photocracking of the surface molecules leaving behind similar fragments on the surface. However, preconditioning of the samples with sputtering, annealing, or activation induces substantial differences in the PY. Sputtering resulted in the most dramatic changes. Annealing of TiZr and St 707 reduced their PY, the latter to the lowest value of all the measured samples. Annealing of either $\mathrm{Pd}$ or Cu-el. samples has no significant effect on their PY. Monochromatic studies, after intense WL irradiation, of the as-received samples have 
shown that the cleaning mechanism induced by photons produces a much less metallic surface than annealing or sputtering. Indeed, most of the sputtered samples and all of the activated getter materials exhibit a Fermi edge, signature of the metallicity of the surface. The reduction of the PY by SR is relevant to the LHC since during a commissioning period, in which the machine will operate with a modified bunch density and possibly bunch spacing, the SR will illuminate the vacuum chamber walls and consequently reduce its PY. The necessary dose and the detailed surface preparation to be used for such a successful conditioning have still to be quantified but the data obtained so far seem to indicate a means to reduce the PY.

The results presented here are directly relevant to the sections of the LHC vacuum system at room temperature that will be illuminated by SR from the arcs, either directly or as a result of multiple reflections. For these sections of the machine the activated getters, St 707 and TiZr, and sputtered $\mathrm{Cu}$ are the best candidate vacuum chamber surfaces in terms of photoelectric emission. To finalize the choice of the beam screen surface, further experiments should be carried out with the sample at cryogenic temperatures (i.e., including beam screen working conditions) where it is expected that cryosorbed molecules will induce strong modifications to the electron emission.

The data presented here have interesting implications for the analysis of electron-stimulated secondary electron emission and the understanding of the electron cloud phenomenon. Assuming similar EDCs for electron induced and the measured photon induced secondary emission, the importance of considering the detailed energy distribution of the emitted electrons is shown. Such an energy distribution is a key parameter in selecting the final vacuum chamber material and its detailed surface preparation. Also it has been noted that an initially performant candidate material may strongly change its emission properties during machine operation due to WL irradiation and/or electron/ion bombardment. This observation, reported for the first time in this context, implies that a careful study of such changes will be necessary in order to guarantee a long term and reliable performance of the machine. Thus, EDCs for electron stimulated secondary electron emission should be studied to provide better input to the understanding of the electron cloud phenomenon.

In addition, CFS spectroscopy has shown that the photon energy dependence of photoemitted electrons is not constant and this energy dependence differs greatly from sample to sample. These adsorption spectra indicate that photon reflection (specular and diffuse) is an important issue for the electron cloud in the LHC which implies that photon energy dependent studies should be made.

Given the scientific objectives, as determined by the electron cloud instability, the cryogenic cooling capacities and the beam lifetime (dominated by the gas density), a measurement protocol should be defined in order to optimize this experimental approach. Also, a devoted experimental setup [22] should be made available and be used also to study other materials (such as $\mathrm{Be}, \mathrm{Al}$, or NEGs) that are proposed in various parts of the machine.

\section{ACKNOWLEDGMENTS}

We gratefully acknowledge the financial support from the INFN-LHCLDS Project, the CERN-INFN agreement No. K434/LHC and No. EC-LSI (HMC12), and BESSY Light Source for access to the TGM7 beam line. We would like to thank C. Benvenuti, P. Chiggiato, and V. Ruzinov for supplying us with TiZr, Cu-el., and Pd samples, R.E. Kirby for supplying us with the TiN coated Al sample, and N. Kos for preparing all the other samples.

[1] J. Gómez-Goñi, O. Gröbner, and A.G. Mathewson, J. Vac. Sci. Technol. A 12(4), 1714 (1994).

[2] O. Gröbner, Vacuum 47, 591 (1996); in Proceedings of the Particle Accelerator Conference, Vancouver, Canada, 1997 (IEEE, Piscataway, NJ, 1998).

[3] O. S. Brüning, CERN LHC Project Report No. 158, 1997.

[4] M. A. Furman, LBNL Report No. LBNL-41482/CBP Note 247, 1998; CERN LHC Project Report No. 180, 1998.

[5] I. R. Collins, A. G. Mathewson, and R. Cimino, in Proceedings of the European Conference on Applications of Surface and Interface Analysis, Göteborg, Sweden, 1997 (Wiley, New York, 1997) (CERN Vacuum Technical Note 97-24, 1997).

[6] J-P. Potier and L. Rinolfi, in Proceedings of the Sixth European Particle Accelerator Conference, Stockholm, Sweden, 1998 (Institute of Physics, Bristol, UK, 1998) (CERN Report No. CERN-PS-98-016-LP, 1998).

[7] Research at BESSY, A User's Handbook, edited by C. Jung (Berliner Elektrononspeicherring-Gesellschaft für Synchrotronstrahlung mbH, Berlin, 1995), 2nd ed.

[8] http://www-cxro.lbl.gov/optical_constants/ and references therein.

[9] M. Krumrey, E. Tegeler, J. Barth, M. Krisch, F. Schäfers, and R. Wolf, Appl. Opt. 27, 4336 (1988).

[10] P. Cruikshank, K. Artoos, F. Bertinelli, J-C. Brunet, R. Calder, C. Campedel, I. R. Collins, J-M. Dalin, B. Feral, O. Gröbner, N. Kos, A. G. Mathewson, L. Nikitina, I. Nikitine, A. Poncet, C. Reymermier, G. Schneider, I. Sexton, S. Sgobba, R. Valbuena, and R. Veness, Proceedings of the Particle Accelerator Conference, Vancouver, Canada, 1997 (IEEE, Piscataway, NJ, 1998) (CERN LHC Project Report No. 128, 1997).

[11] K. Kennedy, B. Harteneck, G. Millos, M. Benapfl, F. King, and R. Kirby, in Proceedings of the Particle Accelerator Conference, Vancouver, Canada, 1997 (IEEE, Piscataway, NJ, 1998) (SLAC Report No. SLAC-PUB7535, 1997) (LBNL Report No. LBNL 39862R1, 1997).

[12] J. Hölzl and F. K. Schulte, in Solid Surface Physics (Springer-Verlag, Berlin, 1979).

[13] Photoemission in Solids, edited by L. Ley and M. Cardona (Springer-Verlag, Berlin, 1979). 
[14] C. L. Foerster, H. Halama, and C. Lanni, J. Vac. Sci. Technol. A 8(3), 2856 (1990).

[15] O. Gröbner, A. G. Mathewson, and P.C. Marin, J. Vac. Sci. Technol. A 12(3), 846 (1994).

[16] C. Benvenuti, P. Chiggiato, F. Cicoira, and Y. L'Aminot, J. Vac. Sci. Technol. A 16(1), 148 (1998).

[17] C. Benvenuti (private communication).

[18] R. E. Kirby (private communication).
[19] O. Gröbner, A. G. Mathewson, P. Strubin, E. Alge, and R. Souchet, J. Vac. Sci. Technol. A 7(2), 223 (1989).

[20] V. Baglin, I. R. Collins, and O. Gröbner, in Proceedings of the Sixth European Particle Accelerator Conference, Stockholm, Sweden, 1998 (Institute of Physics, Bristol, UK, 1998) (CERN LHC Project Report No. 206, 1998).

[21] D. Lawton, CERN EST Technical Report, 1998.

[22] R. Cimino and I. R. Collins (to be published). 